\title{
Molecular magnetism: from chemical design to spin manipulation in molecules, materials and devices
}

\section{E. Coronado}

Instituto de Ciencia Molecular (ICMol). Universitat de Valencia.

E-mail: eugenio.coronado@uv.es

Abstract: In the last 25 years molecular magnetism has been a source of new molecules exhibiting pronounced quantum effects in the single-molecule limit and new multifunctional materials mixing magnetism with a second property such as chirality, photomagnetism, molecular switching or (super)conductivity. At present, this field is rapidly evolving towards the use of these chemically-designed magnetic molecules and materials in other emerging fields including molecular spintronics and quantum computing. In these research crossroads a multidisciplinary approach is pursued in which the molecular magnetism community interacts with the communities working in molecular electronics, spintronics and quantum technologies with the common goals of creating a new generation of spintronic devices and spin quantum bits using molecular materials, or in the longer term one or a few molecules in the race toward miniaturization. From a materials perspective, molecular magnetism is also approaching to two other hot topics of research, namely metal-organic frameworks (MOFs) and twodimensional (2D) materials for which magnetism has only been investigated very recently. In this review I will show the current relevance and future perspectives of molecular magnetism in these four strategic areas of research. 


\section{Historical background}

Molecular Magnetism was born in the 80 's as a result of the transformation of magnetochemistry into an interdisciplinary area, where chemists started a fruitful collaboration with physicists with the goal of designing, synthesizing, characterizing and modelling the magnetic properties of molecular-based compounds. ${ }^{1}$ In this period an initial focus of interest was that of studying simple model systems formed by a reduced number of magnetically-coupled spin centres (mainly homometallic and bimetallic magnetic dimers and small clusters) with the aim of testing the existing solidstate theories about exchange interactions and electron delocalization at the molecular scale. $^{2}$ This work extended in a further step towards the chemical design and physical modelling of low dimensional magnetic materials of increasing complexity (from homometallic chains to bimetallic chain complexes, or to homometallic chains in which the metal ions are bridged by an organic radical, for example). In this context, a major advance in the 80's was the discovery of a novel class of 1D magnetic materials, namely ferrimagnetic chains, formed by two antiferromagnetically coupled spin sublattices alternating along the chain direction. ${ }^{3,4}$ These low dimensional materials motivated the development of new theoretical models with the aim of correlating their distinctive magnetic behaviour - characterized by the presence of a minimum in the magnetic moment at a given temperature, followed by a divergence at lower temperatures - with the values of the two alternating spins and the strength and anisotropy of the exchange coupling between them. In some cases, these 1D materials showed interesting physical phenomena such as slow dynamics of the magnetization ${ }^{5}$ or strong magneto-chiral dichroism. ${ }^{6}$ At the same time, the interchain interactions established between the magnetic chains, which were detrimental for studying the properties arising from the 1D character of the material, resulted to be beneficial to trigger a long-range magnetic order in the crystal below a critical temperature, $\mathrm{T}_{\mathrm{c}}$. Hence, this coordination chemistry approach was soon exploited in the 90's to design magnetic materials exhibiting spontaneous magnetization below $\mathrm{T}_{\mathrm{c}}$ (molecule-based magnets). Together with other chemical approaches, ${ }^{7}$ including organic chemistry, organometallic chemistry and solid-state chemistry, this coordination chemistry approach has provided since then a plethora of new magnets with different topologies and dimensionalities ${ }^{8,9,10}$ and with $\mathrm{T}_{\mathrm{c}}$ values reaching room temperature. ${ }^{11}$ 
In parallel with this development, the magnetochemical community was also fascinated by metal complexes undergoing a spin transition near room-temperature and by the possibility of tuning such a molecular bistability through physical stimuli such as temperature, light or pressure. ${ }^{12,13}$ Very soon this phenomenon opened the possibility to develop switching materials of direct application in several technological areas such as molecular or pressure sensing, ${ }^{14}$ actuators, ${ }^{15}$ and thermal displays. ${ }^{16}$

In summary, the appearance of Molecular Magnetism motivated chemists for using magnetism not only as a tool to correlate the structure and bonding of a given molecule with the presence of unpaired electrons, but also for taking advantage of this fundamental understanding to design novel crystalline molecule-based materials exhibiting new physics and/or useful magnetic properties, similar to those found in the inorganic materials (ferromagnetism, for example), but with some specific and distinctive features and additional possibilities (optical transparency, insulating character, low density, preparation via solution techniques, photomagnetism, etc.).

After this initial period, Molecular Magnetism grew very fast to consolidate into a field in which chemists were designing more and more complex materials and physicists were interested in the observation of new types of magnetic phenomena in these materials. ${ }^{17}$ In this period, which covers the last 25 years or so, two different classes of magnetic materials have been dominating the field: the so-called single-molecule magnets and the multifunctional magnetic materials.

The first class of materials — single-molecule magnets (in short SMMs) — opened up a brand new research field since for the first time it was possible to observe quantum effects in mesoscopic magnets. At that time physicists were looking for small magnetic particles, all identical to each other, in order to test theories that suggested that it was possible to observe quantum effects in these nanoparticles. The main difficulty was that of obtaining assemblies of identical particles. The chemists solved this problem by using a molecular approach, which allowed them to prepare identical magnetic clusters and to assemble them in a crystalline lattice. Thus, in 1993 crystals formed by a cluster comprising twelve manganese ions - the so-called $\mathrm{Mn}_{12}$ acetate- were prepared and magnetically characterized. These molecular systems showed a large magnetic hysteresis at low temperatures (below $2 \mathrm{~K}$ ) similar to that observed in hard magnets, but of molecular origin. ${ }^{18}$ This discovery suggested that indeed single molecules of nanometer size may behave as tiny magnets being able to store information. Soon after 
this discovery, in 1996, it was also shown that crystals of these molecules exhibited quantum tunnelling effects. ${ }^{19,20}$ This last phenomenon was acknowledged as one of the 23 milestones in the study of spin of the $20^{\text {th }}$ century in the special collection "Milestones in Spin" published by Nature in March 2008. ${ }^{21}$

The second class of materials - multifunctional materials - impacted not only in Molecular Magnetism but also in other areas of the Materials Science research, as for example in that focused on molecular conductors and superconductors. In fact, these molecular-based materials enabled to combine in the same crystalline solid two electronic properties of technological interest (superconductivity and ferromagnetism, for example) that, at that time, were difficult or impossible to achieve in a purely inorganic solid. ${ }^{22}$ A variety of crystalline molecular materials exhibiting co-existence or even coupling of two properties were reported in the period around the end of the $20^{\text {th }}$ century and the beginning of the $21^{\text {th }}$ century as, for example, the design of molecular conducting magnets, ${ }^{23}$ chiral magnets, ${ }^{24,25}$ porous magnets ${ }^{26}$ or stimuli-responsive magnets. $^{27,28,29,30}$

Nowadays these two classes of molecular materials still concentrate most of the attention in Molecular Magnetism. However, in this last period Molecular Magnetism has evolved towards the use of these chemically-designed magnetic molecules and materials in other emerging fields including molecular spintronics and quantum computing. In these research crossroads a multidisciplinary approach is pursued in which the molecular magnetism community interacts with the communities working in molecular electronics, spintronics and quantum technologies with the common goals of creating a new generation of spintronic devices and spin quantum bits (qubits in short) using molecular materials, or in the longer term one or a few molecules in the race toward miniaturization. From a materials perspective, Molecular Magnetism is also approaching two other hot topics of research, namely that of the metal-organic frameworks (MOFs) and that of the two-dimensional (2D) single-layer materials for which magnetism still remains an elusive property. In this article I will show the current relevance and future perspectives of Molecular Magnetism in these four strategic areas of research. 


\section{Molecular spintronics}

Spin-based electronics — which aims to use the spin of the electron for information processing - is one of the emerging branches in nanotechnology and the most active area within nanomagnetism. So far this area has been almost exclusively based on conventional inorganic metals and semiconductors. However, molecules show specific properties that make them optimal for their integration into spintronic structures such as exceptionally long spin relaxation times and pronounced quantum effects in the singlemolecule limit. ${ }^{31,32,33}$ Hence, the use of molecules in spintronics can foster new opportunities in this area both in terms of new concepts and in terms of new performing materials and devices. The first report showing the ability of an organic semiconductor (alpha-sexithienyl, $\mathrm{T}_{6}$ ) to transport a spin polarized current was published ${ }^{34}$ in 2002; soon after, in 2004, this spin polarization was demonstrated in a spin valve device formed by the integration of a nanometric-thick layer of (non-magnetic) molecules of $\mathrm{Alq}_{3}$ (tris (8-hydroxy-quinoline) aluminium (III) complex) in between two ferromagnetic electrodes. ${ }^{35}$ The structure of these molecular spin valves is similar to that schematized in Box 1 for a conventional spin valve. These pioneering reports constituted the starting point of the molecular spintronics field (initially known as organic spintronics, or molecule-based spintronics). ${ }^{36}$ More recently, organic spintronics has also evolved towards other challenging goals such as the design of multifunctional spintronic devices, as for example, spin-OLEDs ${ }^{37,38}$ and molecular spin-photovoltaic devices. ${ }^{39}$ The idea behind these devices is to enhance their efficiencies by controlling the electron-hole pair recombination via a spin polarization of the charge carriers induced by the magnetic electrodes. On the other hand and thanks to the previous advances in molecular electronics, the fabrication of nanospintronic devices incorporating one or a few molecules (not necessarily organic) in between the electrodes has become a hot topic in recent years as well.

In these two topics —organic spintronics and molecular nanospintronics- Molecular Magnetism can play a prominent role as it offers the possibility of incorporating in these devices a variety of magnetic molecules undergoing interesting effects such as quantum tunnelling of the magnetization (in case of SMMs), spin switching (in case of spin crossover complexes), or, simply, spin filtering owing to their magnetic nature. From a chemical point of view, this crossroad involves a new twist in Molecular Magnetism since the focus of interest has evolved from the chemical design of crystalline 
materials towards the processing of these magnetic molecules and materials at the nanoscale to afford thin films, self-assembled nanostructures or individual molecules. It is worth noting that the use of magnetic molecules in this context is very recent. In fact, in a typical molecular spintronic device the magnetic component is provided by the ferromagnetic electrodes that are often used as exclusive source of spin polarization, while non-magnetic molecules are used to tailor the interaction and energy landscape of the molecular/inorganic hybrid interface, and to collect and transport the spins.

Obviously, a prior condition for integrating these molecules into devices requires a detailed study of their deposition, positioning, self-assembly and characterization on various surfaces in order to check their stability and properties under these circumstances. Some significant results in this context have been the observation of a magnetic hysteresis in SMMs deposited on gold ${ }^{40}$ or on an insulating $\mathrm{MgO}$ thin layer, ${ }^{41}$ the positioning of SMMs with nanometric accuracy on silicon surfaces, ${ }^{42}$ the reversal of the spin polarization in phtalocyanine molecules deposited on magnetic surfaces, ${ }^{43}$ or the observation of spin filtering effects in magnetic complexes deposited on gold, ${ }^{44}$ just to mention a few. This aspect has centred a lot of effort in the last fifteen years or so, fostering a close collaboration between the magnetic community and the molecular electronics community. In addition, it has required, and still requires, a strong chemical effort in order to design molecular coordination complexes that survive when they are in direct contact with the device components (electrodes and substrates), while being compatible with the processing techniques used in spintronics. These chemical concerns are a serious limitation since, in contrast to what happens with organic molecules, these coordination complexes are often chemically unstable in presence of a metallic substrate (since they undergo redox reactions) and furthermore, they are not always compatible with the high-vacuum technologies typically used in spintronics (since in most cases they are forming salts that cannot be sublimed). In fact, the number of thermally stable magnetic complexes that have been experimentally probed to be suitable for molecule-based spintronics remains very reduced. In the following, the role played by these magnetic molecules in spintronics will be illustrated with some examples.

Organic spintronics. Dealing with the integration of magnetic molecules into spin valve devices, the magnetic analogues of the $\mathrm{Alq}_{3}$ molecule constitute an interesting 
class of magnetic molecules. A first example is provided by the trimeric rare-earth quinoline complexes formulated as $\operatorname{Ln}_{3} \mathrm{q}_{9}$ (Figure 1 top). From a chemical point of view, these molecules were chosen in view of their ability to form stable and uniform films upon sublimation and deposition onto ferromagnetic surfaces via ultra-high vacuum (UHV) deposition techniques. From a magnetic point of view, these lanthanoid complexes are very versatile as they are able to host trivalent lanthanoid ions having high magnetic moments $\left(\mathrm{Tb}^{3+}, \mathrm{Gd}^{3+}\right.$ or $\left.\mathrm{Dy}^{3+}\right)$, but also zeronet moment $\left(\mathrm{Y}^{3+}\right.$ derivative). Spin valves based on these complexes were fabricated to test the effect of the magnetic moments on the spin-polarized injection and transport properties of these devices. Thus, $\mathrm{Tb}_{3} \mathrm{q}_{9}$ and $\mathrm{Y}_{3} \mathrm{q}_{9}$ molecular layers were inserted in between permalloy $(\mathrm{NiFe})$ and Co ferromagnetic electrodes. ${ }^{45}$ In both devices a sizeable magnetoresistance was observed at room temperature down to a molecular thickness of $15 \mathrm{~nm}$. This result suggests that the spin injection is barely affected by the magnetic nature of the molecule. This also indicates that the spin transport properties are mainly governed by the quinoline ligand states, being largely insensitive to the magnetic moment of the central metal ion. Similar results have been observed in spin valve devices containing as interfacial layers organic radicals ${ }^{46}$ or double-decker bis-phtalocyaninato lanthanoid complexes, $\mathrm{LnPc}_{2}{ }^{47}$

A second example is provided by the mononuclear lanthanoid quinoline complexes of general formula $\mathrm{A}^{+}\left[\mathrm{Ln}\left(5,7 \mathrm{Cl}_{2} \mathrm{q}\right)_{4}\right]^{-}$, with $\mathrm{Ln}=\mathrm{Y}^{3+}, \mathrm{Tb}^{3+}$ and $\mathrm{Dy}^{3+}$ and $\mathrm{A}^{+}=\mathrm{Na}^{+}, \mathrm{NEt}_{4}{ }^{+}$ and $\mathrm{K}_{0.5}\left(\mathrm{NEt}_{4}\right)_{0.5}{ }^{+}$(Figure 1 top). Surprisingly, the sodium salts of these anion complexes (in short $\mathrm{NaLnClq}$ ) can also be sublimed and deposited on various substrates, including ferromagnetic surfaces, while maintaining intact both their molecular structures and their magnetic behaviour. ${ }^{48}$ In this case, highly homogeneous ultrathin films (10 $\mathrm{nm}$ thick) of these molecules can be deposited on metallic ferromagnetic substrates, thus allowing us to fabricate molecular spin valves. Interestingly, a sign-inversion in the magnetoresistance of the device can be achieved as a consequence of specific molecule-to-metal hybridization at the molecular/ferromagnetic interface ${ }^{49}$ (Figure 1 bottom). This capacity of an interfacial molecular layer to modify or even to invert the spin-polarization of the current extracted from a ferromagnetic electrode — known as 'spinterface' effect— ${ }^{50,51}$ is probably the most appealing result found in spin valve devices based on molecules, an effect that is very complicated or impossible to achieve with inorganic materials. In the present 
example, one observes that when the NaDyClq molecule is combined with Co and $\mathrm{NiFe}$ electrodes, the magnetoresistance is positive if an $\mathrm{AlO}_{\mathrm{x}}$ barrier is inserted between $\mathrm{NiFe}$ and the molecule, but it changes to negative when the $\mathrm{AlO}_{\mathrm{x}}$ layer is placed on the Co electrode. This result has been attributed to the formation of specific hybrid electronic states at the molecular/NiFe interface, which affords a negative spin polarization, while at the molecular/Co interface the mixing is weaker and the spin polarization stays positive.

This part has emphasized that molecules (not necessarily magnetic) in close proximity with the ferromagnetic electrodes can be used not only as spin transport media of spintronic devices but also for tuning the spin polarization of ferromagnetic materials at the interface. ${ }^{52}$ Still, magnetic molecules have played a very limited role yet. Thus, the magnetic functionality of molecules and their most interesting properties (slow relaxation of the magnetization, quantum tunnelling effects, spin switching, etc.) remain fully unexploited.

Molecular nanospintronics. A current trend in spintronics is to work in reduced dimensions with the aim of discovering/exploiting new magnetic (quantum) phenomena and of manipulating spins one by one. The study of the single nano-objects connected to two magnetic electrodes has been limited to inorganic nanoparticles and organic carbon nanostructures. Although the transport through single molecules has been extensively studied in molecular electronics, very few experiments have been reported so far in the case of magnetic molecular systems, including single magnetic molecules and molecular-based magnetic nanoparticles. In contrast to what happens in organic spintronics, in molecular nanospintronics magnetic molecules based on coordination chemistry constitute a key element of the device. Two methods are commonly used to fabricate these devices. In one case, the molecule is connected through a STM tip and a conducting surface; the other case involves a single-molecule transistor architecture in which a break-junction setup traps the molecule between two electrodes, while a third electrode that acts as a gate, may be incorporated. Still, many problems, which are intrinsic to the magnetic molecules, need to be solved to obtain reliable results. A first issue deals with the stability of these molecules when they are deposited on, or contacted to, a metallic surface. In fact, as mentioned above, most of the magnetic molecules of interest are coordination metal complexes, which often undergo important structural and chemical changes under these drastic conditions. A second issue is that of 
positioning an individual magnetic molecule in a controlled manner on a surface (or in between two electrodes). This often requires the functionalization of the molecule in order to tune its interaction/orientation with the electrodes.

In view of these chemical and processing challenges, one can understand why the theoretical predictions are ahead the experimental achievements. However, thanks to the close interaction between chemists - able to design robust magnetic molecules which can survive when contacted to electrodes - and physicists and engineers - able of using nanotechnologies in order to fabricate devices with enhanced sensitivities and reliabilities - the first successful experiments on functional nanodevices based on single-magnetic nanoobjects have recently been performed, as is illustrated below using spin-crossover (SCO) nanoparticles or magnetic molecules as source of spins. This joint venture will enable the creation of electrically addressable single-molecule spin switches and magnetic qubits, opening the possibility to tailor and manipulate molecules down to the single spin.

In the first example, the electrical addressing of a molecular spin is achieved in a SCO nanoparticle placed in a break-junction setup. In bulk, the SCO systems undergo a switching from low-spin to high-spin upon heating. This electronic spin transition is often accompanied by a thermal hysteresis at relatively high temperatures, a property that can be used for memory applications in electronic devices. In the experiment, a single-nanoparticle of a SCO material of ca.10 $\mathrm{nm}$ has been placed in between two gold electrodes separated by a $5 \mathrm{~nm}$ gap (Figure 2 left). The nanoparticles of choice are those based on the polymeric one-dimensional $\mathrm{Fe}^{+2}$ compound $\left(\left[\mathrm{Fe}(\mathrm{Htrz})_{2}(\operatorname{trz})\right]\left(\mathrm{BF}_{4}\right)\right),(\mathrm{Htrz}=$ 1,2,4-triazole), which preserve this memory effect. The transport properties of this device have shown to be sensitive to the spin transition; thus, a thermal hysteresis loop in the electrical conductance near room temperature has been observed (Figure 2 left), with a ratio of $\approx 3$ between the low conductance state (associated to the low spin state) to the high conductance state (associated to the high spin state). ${ }^{53}$ Interestingly, this spin switching can also be triggered by applying a bias voltage, at constant temperature. Hence, this device can allow us to electrically read-out and switch the molecular spin at the nanoscale. In a further experiment, it has been shown that the device performance can be sharply improved -with ON/OFF switching ratios of the conductance increasing up to ca. 300 -if instead of putting a single nanoparticle in the gap, one 
places 2D assemblies of these SCO nanoparticles ${ }^{54}$ - or to ca. 5000 in assemblies of $\mathrm{Au} @$ SCO core-shell nanoparticles. ${ }^{55}$

This electrical detection and manipulation of the electronic spin at the nanoscale (and near room temperature) opens the way towards the use of these switching nanoparticles as bits of memory. Still, the low thermal stability of these SCO nanoparticles at the temperatures where the spin transition occur $(\approx 360 \mathrm{~K})$ strongly limits this application. This problem has been solved by chemical means via the coating of these nanoparticles with a nanometre-thick shell of silica, which sharply improves the stability of the nanoparticles upon repeated thermal cycling. ${ }^{56}$

In the second example, electrical read-out and manipulation of the spin have been achieved at the single-molecule level. In this case a conveniently functionalized SMM based on a bis-phtalocyanine $\mathrm{Tb}^{+3}$ complex, $\mathrm{TbPc}_{2}$, has been embedded in an electronic circuit (Figure 2 right). ${ }^{57}$ In this robust magnetic molecule, the molecular environment around $\mathrm{Tb}^{+3}$ leads to an anisotropic ground electronic state $m_{J}= \pm 6$, which is strongly coupled to its nuclear spin, $I=3 / 2$, causing a splitting in the resulting electron-nuclear spin states according to the four nuclear spin components $m_{I}(3 / 2,1 / 2,-1 / 2$ and $-3 / 2)$. In this system the transport occurs through the $\pi$-system of the phtalocyanine ligands, which contain a delocalized unpaired electron exchange-coupled to $\mathrm{Tb}^{+3}$. Due to this coupling, the transport properties can be influenced by the magnetic properties of $\mathrm{Tb}^{+3}$ and therefore, they are expected to be sensitive to its nuclear spin state. This is precisely what is observed in the magneto-transport measurements, which show abrupt changes in the conductance for some specific values of the magnetic field, which can be associated to specific values of the nuclear spin states. In fact, at each one of these fields two electron-nuclear levels having the same nuclear spin component and opposite magnetic electronic states are crossing; thus, at these anti-crossing points the molecule can reverse its magnetic moment from $m_{J}=-6$ to $m_{J}=+6$ via quantum tunnelling, while keeping its nuclear spin state (Figure 2 right). By playing with the magnetic field and with radiofrequency pulses it has been shown that this nuclear spin can be coherently manipulated to perform quantum operations, ${ }^{58}$ thus illustrating the power of magnetic molecules as qubits in quantum information technologies, ${ }^{59}$ a topic that will be discussed in the next section. Obviously, all these measurements have required very low temperatures (ca. $0.08 \mathrm{~K})$. 


\section{Molecular spins for quantum technologies.}

An appealing possibility arising from the quantum effects associated to the above spintronic nanodevices is that of using magnetic molecules not only for the storage of classical bits, but also for the creation, manipulation and readout of quantum superpositions of two spin states $|0\rangle$ and $|1\rangle$, thus providing practical realizations of spin qubits, which are the basic units of future quantum computers (Box 2). We are still far from reaching this final goal, since these quantum superpositions are extremely fragile in real systems, especially in the solid state, losing rapidly the quantum information. This effect, known as decoherence, together with the difficulty in creating scalable networks of spin qubits able to interact and implement quantum algorithms, are the two major obstacles encountered in the fabrication of solid-state quantum devices. In this context, a molecular approach can compete with, or even be advantageous to a conventional approach, which is based on extended inorganic lattices. Thus, the versatility of chemistry can be exploited to adjust the spin environment in order to minimize decoherence. At the same time, molecules are unmatched in terms of scalability since they are the quantum objects with the highest capacity to be replicated in large numbers and arranged via self-assembly and molecular recognition processes. These features have attracted the interest of the Molecular Magnetism community, which have started to investigate the properties of molecular coordination complexes as possible candidates of spin qubits. In fact, some of the research on SMMs has now been re-directed towards their use in quantum computation. This is the case of magnetic polyoxometalates (POMs) based on lanthanides, which under some circumstances can either behave as SMMs or as molecular spin qubits. ${ }^{60}$ Note that the integration of molecular spin qubits into scalable devices, which is the final goal of this research, remains largely unexplored. At present, most of the attention is still focussed on the previous steps, that is the design of robust spin qubits and their coupling to give spin quantum gates. Here the landmarks conquered by Molecular Magnetism in quantum technologies will be illustrated through some relevant examples. Recent accounts that discuss this topic in more detail can be found in refs. 59 and 61.

As far as the design of qubits is concerned, molecular spin qubits with large quantum coherences have been reported (see below) by chemically controlling the decoherence sources. Thus, magnetic noise can be minimized through the use of magnetically diluted samples and nuclear spin-free ligands and solvents. More 
complicated is to control the couplings of the spins with the vibrations. Here the aim is that of identifying the vibrational modes that are most detrimental to quantum coherence in each structure in order to suppress them by chemical optimization. ${ }^{62}$ Examples of robust (coherent) molecular spin qubits include the tris-dithiolate vanadium complex $\left[\mathrm{V}\left(\mathrm{C}_{8} \mathrm{~S}_{8}\right)_{3}\right]^{2-},{ }^{63}$ the $\left[\mathrm{Cu}(\mathrm{mnt})_{2}\right]^{2-}\left(\mathrm{mnt}^{2-}=\text { maleonitriledithiolate }\right)^{64}$ and the vanadyl phthalocyanine complex ${ }^{65}$ (see Box 2). In frozen solution and under optimized conditions of temperature, magnetic dilution and isotopic purity in order to minimize the magnetic noise, the first one exhibits $T_{2}$ values in the millisecond range at low temperature. On its turn, the last example presents $T_{2}$ values up to a microsecond at room temperature thanks to the optimization of the molecular vibrations. These coherence times are quite competitive with those reported for spin-qubits isolated in inorganic lattices, such as phosphorous impurities in silicon (with $\mathrm{T}_{2}$ values of the order of seconds at low temperature) and diamond nitrogen-vacancy (NV) centres (with $\mathrm{T}_{2}$ values in the millisecond range at room temperature). However, these high coherence times have been obtained in magnetically diluted samples only. At this point, a fundamental paradox, between reducing decoherence by diluting spin qubits in a diamagnetic matrix and coupling these spin qubits in order to manipulate quantum information, appears.

A trick to overcome this problem is based on the engineering of the energy level structure of the molecule in such a way as to make the spin qubit insensitive to magnetic noise (i.e., invisible to a magnetic field). ${ }^{66}$ This requires the mixing of the qubit states in order to generate an avoided level crossing (or tunneling gap, $\Delta$ ) upon variation of the applied magnetic field (Figure 3 top). At this optimal operating point —or atomic clock transition - the spin qubit dynamics becomes protected against dipolar decoherence, leading to an enhancement in $\mathrm{T}_{2}$. This approach is illustrated in the anion $\left[\mathrm{HoW}_{10} \mathrm{O}_{36}\right]^{9-}$, in short $\mathrm{HoW}_{10}$, which contains a $\mathrm{Ho}^{3+}$ ion encapsulated in between two polyoxometalate moieties (Figure 3 top). In this molecular nanomagnet unusually long coherence times are found at the four clock transitions which are present in its electronic structure ( $\mathrm{T}_{2}$ up to $8.4 \mu \mathrm{s}$ at $5 \mathrm{~K}$ ), even in concentrated samples, thanks to the presence of a large tunneling gap, $\Delta \sim 0.3 \mathrm{~cm}^{-1}$, in the ground spin doublet $\pm \mid 4>$ (Figure 3 top) Hence, this molecular approach provides a source of chemically designed spin qubits with enhanced coherences thanks to the control in their chemical composition, electronic structure and environment around the magnetic metal ion. 
With respect to the second step, chemistry can be exploited to design molecules hosting more than one spin qubit, which can enable the realization of simple quantum algorithms. In Box 2 (middle) some candidates of these molecular quantum gates are plotted. A first example of quantum gate has been provided by the vanadyl-bicapped reduced Keggin POM, where electrons injected into the central Keggin cluster via an STM tunnelling current are expected to control the exchange coupling between the vanadyl spins. ${ }^{67} \mathrm{~A}$ similar idea has been proposed by assembling molecular $\left\{\mathrm{Cr}_{7} \mathrm{Ni}\right\}$ rings, which act as the qubits, into structures suitable for either the CNOT or $\sqrt{\text { iSWAP }}$ gates. ${ }^{68}$ Very often the magnetic ions of choice have been lanthanide ions since they lead to weak exchange interactions that are compatible with the microwave energies provided by EPR spectroscopy, something that is difficult to achieve with transition metals. A simple system of this kind consists of an unsymmetrical dinuclear complex LnLn' exhibiting two coordination sites, which are able to host distinguishable qubits that can be addressed separately using microwave pulses. ${ }^{69}$ Still, this dinuclear magnetic unit is difficult to keep intact upon dilution with non magnetic lanthanoid ions. Hence, this approach is limited by the magnetic noise arising from dipolar interactions between the magnetic ions. This difficulty may be overcome by integrating several qubits in a single magnetic ion as in this case the magnetic dilution is trivial. This can be achieved when the ground spin state of the magnetic ion is a multiplet instead of a doublet. This multilevel system, termed as qudit, has $\mathrm{d}=2^{\mathrm{N}}$ levels and allows in principle to realize $\mathrm{N}$ qubits, thus enhancing the density of quantum information that can be handled with molecular systems. For electron spins, this approach has been illustrated in a single crystal constituted by a "doughnut" shaped POM molecule formulated as $\left[\mathrm{Gd}\left(\mathrm{H}_{2} \mathrm{O}\right) \mathrm{P}_{5} \mathrm{~W}_{30} \mathrm{O}_{110}\right]^{12-}$ (in short $\mathrm{GdW}_{30}$ ) (see Box 2). In this molecular nanomagnet the environment around the nearly-isotropic $\mathrm{Gd}^{3+}$ ion has been designed in order to afford an overall energy splitting of the $S=7 / 2$ multiplet well below $1 \mathrm{~cm}^{-1}(30 \mathrm{GHz})$. Such a small splitting has allowed to detect through continuous $\mathrm{X}$-band EPR $\left(9.8 \mathrm{GHz}=0.33 \mathrm{~cm}^{-1}\right)$ the seven allowed transitions connecting the $2 \mathrm{~S}+1=$ 8 spin states, then to address them separately by tuning the magnetic field and, finally to coherently manipulate them by using pulsed EPR on single crystals (Figure 3 bottom). ${ }^{70}$ Therefore, this molecule provides an experimental realization of a $\mathrm{d}=8$ qudit, which is equivalent to a quantum gate formed by three addressable qubits. Nuclear spins have been also useful to illustrate this qudit approach. Thus, the $\mathrm{TbPc}_{2}$ complex reported in the previous section provides an example of a $d=4$ qudit as it has a nuclear spin 
quadruplet $I=3 / 2$ with unevenly spaced levels that result from the combination of hyperfine and nuclear quadrupolar interactions. This is equivalent to two addressable qubits that can be manipulated by microwave pulses. It has enabled the realization of a quantum algorithm (Grover's search algorithm) on a single molecule. ${ }^{59}$

A general conclusion of this part is that the recent advances in this area have often involved the study of crystalline samples based on well-known magnetic complexes (mononuclear lanthanoid complexes in particular). Clearly, things will become much more challenging when the objective is to integrate these molecular spin qubits into scalable devices. In fact, this last step requires to go from the single crystal to the single molecule in order to implement a scalable architecture in which individual spin qubits are coherently wired up. Superconducting coplanar resonators have been proposed to wire up these molecular spin qubits via the individual photons confined in the chip. In this chip a single microwave photon can introduce an effective coupling between any pair of qubits, even at large distances, since its wavelength is very large (in the range 3 to $30 \mathrm{~mm}$ ) (Box 2 bottom). In order to implement quantum gates between these two qubits, the spin-photon coupling has to be sufficiently strong as compared to decoherence times. To maximize this coupling the first condition is to position the molecules in nanoconstrictions of the superconducting transmission lines since this leads to an enhancement in the magnetic field generated by the photon. Hence, deposition techniques able to place the molecules with nanometre accuracy in these specific positions need to be used. On the other hand, a deep knowledge of the electronic structure of the spin qubit is required, as the strength of this spin-photon coupling has been predicted to depend on the total angular momentum of the molecule, $\mathrm{J}$, and on its magnetic anisotropy, ${ }^{71}$ being maximized either in molecules having an easy plane anisotropy (i.e., a ground doublet $m_{J}= \pm 1 / 2$ ) coming from a $\mathrm{J}$ value as large as possible, or in molecules having a clock transition between tunnel split $\pm m_{J}$ states, with the highest possible $m_{J}$. Notice that some of these electronic requirements are just opposed to those required to design robust SMMs, which involves large superparamagnetic barriers for the magnetization reversal. This requires an easy axis anisotropy, i.e., a ground doublet with a large $m_{J}$ value) and tunnelling gaps within the \pm $m_{J}$ ground doublet as small as possible. Hence, many magnetic molecules that have remained so far ignored in the SMM context, could now become of prior interest in the quantum information context. 


\section{Magnetic MOFs.}

Porous coordination polymers (also known as MOFs, Box 3) have recently emerged as a tuneable platform for designing functional materials with promise for applications in a number of areas including gas storage/separation, energy conversion/storage, molecular sensing, drug delivery and catalysis. ${ }^{72}$ So far, porosity and chemical stability have been the two properties of MOFs that have concentrated most of the attention. In turn, the electronic properties of these crystalline materials have been loosely investigated. Thus, only very recently the first examples of conducting MOFs, ${ }^{73,74,75,76}$ luminescent MOFs ${ }^{77,78}$ or magnetic MOFs $s^{79,80}$ have been reported.

One of the reasons that justify the interest for magnetic MOFs is the possibility to generate multifunctional materials in which the magnetism can be tuned by the presence of molecules in the pores. This may be of interest for sensing the molecular species trapped in the pores through their magnetic response. However, a major obstacle is that porosity and cooperative magnetism are two inimical properties that require opposite structural features (large porosity requires the use of long organic linkers between the metal centres, while strong exchange interactions require the use of short ones). In fact, this "magnetic exchange" approach is limited by the relatively low temperatures at which the cooperative magnetism appears (typically much below $100 \mathrm{~K}$ ).

To overcome this limitation, a second procedure based on the integration of "spincrossover complexes" at the nodes of the framework can be undertaken as the spin transition typically occurs near room temperature. In that case, cooperative effects are controlled by elastic forces in the lattice, which can be maintained at long metal-metal distances thanks to the polymeric nature of the MOF lattice. An illustrative example of this procedure is provided by the coordination polymers $\left[\mathrm{Fe}(\mathrm{btzx})_{3}\right]\left(\mathrm{ClO}_{4}\right)_{2}$ and $\left[\mathrm{Fe}(\mathrm{btzx})_{3}\right]\left(\mathrm{BF}_{4}\right)_{2}$, where btzx stands for the bistetrazol- $p$-xylene ligand (Figure 4 top). These compounds form chain structures formed by the connection of $\mathrm{Fe}^{2+}$ centres to three btzx ligands in syn conformation and contain internal cavities of nanometric size. ${ }^{81}$ They exhibit a cooperative spin transition centred at $\mathrm{T}_{1 / 2} \approx 200 \mathrm{~K}$ with a thermal hysteresis of $4 \mathrm{~K}$. As MOFs, they are able to trap gas molecules. For example, it has been found that they selectively trap $\mathrm{CO}_{2}$ from a mixture containing $\mathrm{CO}_{2}$ and $\mathrm{N}_{2}$. The presence of $\mathrm{CO}_{2}$ in the cavity can be detected by a shift in the spin transition temperature, $\mathrm{T}_{1 / 2}$, by $5 \mathrm{~K}$ (Figure 4 top). Other gases like $\mathrm{CH}_{4}, \mathrm{C}_{2} \mathrm{H}_{4}$ or $\mathrm{C}_{2} \mathrm{H}_{2}$ can also 
been trapped into the cavities. These gases affect differently the spin transition depending on the strength of the interaction. Thus, whereas loading of $\mathrm{CO}_{2}$ gas molecules induces an increase of $5 \mathrm{~K}$ of the $\mathrm{T}_{1 / 2}$, sorption of ethylene causes the opposite response from the framework, i.e. a small reduction in the transition temperature, and sorption of methane, ethane and $\mathrm{CO}$ do not affect the transition temperature. ${ }^{82}$ This different behaviour is related to the gas-framework interaction, which is stronger for the $\mathrm{CO}_{2}$.

Another family of SCO MOFs able to trap molecules is the so-called Hoffmann clathrates. In this family the effect on the spin transition of different guests incorporated in the pores has been extensively studied. ${ }^{83}$ For example, it has been demonstrated that chemisorption of $\mathrm{SO}_{2}$ molecules stabilizes the low spin state of the $\mathrm{Fe}^{2+}$ ions and causes an increase of $8 \mathrm{~K}$ in the transition temperature (Figure 4 bottom). ${ }^{84}$

So far these sensing studies have been performed on crystalline bulk samples. Still, detailed studies of this molecular sensing should require in the future of an appropriate processing of the materials to optimise the detection / separation of these molecular species. Efforts in this context have just started to be developed, as for example the preparation of ultrathin films of MOFs on surfaces (SURMOFs) and their integration in electronic devices. ${ }^{85,86,87}$

A second reason that justifies the interest of MOFs in molecular magnetism arises from the possibility offered by these coordination polymers to generate magnetic nanostructures well organized and insulated in the space thanks to their structural features. This control over the topology and separation between the magnetic centres may be useful for generating non-trivial interactions and scalable spin structures. This feature offers improved capabilities from the point of view of quantum information compared to zero-dimensional systems. It has been recently exploited in the family formulated as $\mathrm{Ln}(\text { bipyNO})_{4}(\mathrm{TfO})_{3}$ (bipyNO = 4,4'-bypyridyl-N,N'-dioxide, $\mathrm{TfO}=$ triflate). ${ }^{88}$ Its structure consists of a $3 \mathrm{D}$ coordination network containing a regular arrangement of three orientationally different lanthanoid single-ion magnets (SIMs) placed at the nodes of a porous cationic framework formed by bipyNO as bridging ligand (Figure 5 top). The controlled organization of SIMs having three crystallographically different metal sites in this open framework offers novel possibilities in terms of scalability since the spins of these distinct metal sites can be addressed independently using pulsed EPR spectroscopy. 
The above examples have exploited the ability of these materials to incorporate the magnetism at the nodes of their frameworks (approach (i) in Box 3). Still, the pores offered by these coordination polymers can allow us to fill them with magnetic molecules (approach (ii) in Box 3). This has been recently explored by inserting into the pores several single-molecule magnets ${ }^{89}$ and spin crossover molecules. ${ }^{90}$ When the functional properties are also present in the framework, this hybrid approach does provide ideal platforms to create new multifunctional materials covering from the simple co-existence of different properties - coming from the framework and the magnetic guest molecule - to a synergy between these functionalities as a result of the coupling between the two properties (approach (iii) in Box 3). Although very rare, this last situation has been encountered in the hybrid compound formulated as $\left[\mathrm{Mn}^{\mathrm{III}} \text { (salen) }\left(\mathrm{H}_{2} \mathrm{O}\right)\right]_{2}\left[\mathrm{Mn}^{\mathrm{II}} \mathrm{Cr}^{\mathrm{III}}(\mathrm{ox})_{3}\right]_{2} \cdot(\mathrm{MeOH}) \cdot\left(\mathrm{CH}_{3} \mathrm{CN}\right)_{2}$, which is formed by the SMM $\left[\mathrm{Mn}^{\mathrm{III}} \text { (salen) }\left(\mathrm{H}_{2} \mathrm{O}\right)\right]_{2}{ }^{2+}$ (salen ${ }^{2-}=\mathrm{N}, \mathrm{N}^{\prime}$-ethylenebis-(salicylideneiminate) $)$ inserted into a ferromagnetic 3D oxalate network $\left[\mathrm{Mn}^{\mathrm{II}} \mathrm{Cr}^{\mathrm{III}}(\mathrm{ox})_{3}\right]^{-91}$. Interestingly, the presence of a magnetic coupling between the soft ferromagnetic $\mathrm{MnCr}$ lattice with the highly anisotropic spin lattice provided by the SMM, leads to a drastic enhancement of the coercive field of the hybrid (from 10 to ca. $800 \mathrm{G}$ ), which now behaves as a permanent magnet below $5 \mathrm{~K}$ (Figure 5 bottom). This behaviour resembles that found in alloys of $3 \mathrm{~d}$ metals and lanthanide ions that, like $\mathrm{NdFe}_{14} \mathrm{~B}$, are among the best permanent magnets known to date. In that case the enhancement in the magnetic coercivity is due to the coupling between a highly anisotropic magnetic component (the lanthanoid) and a soft magnet (the $3 \mathrm{~d}$ metal extended lattice). In the example reported here, the magnetic anisotropy is provided by a SMM cluster based on a d-transition metal ion, thus opening an alternative approach to design lanthanoid-free permanent magnets.

\section{D magnetic materials.}

Since the isolation of graphene in 2004, other atomically thin layers (aka. 2D materials) have emerged, which cover a wide range of electronic and magnetic properties and chemical compositions (Box 4). Notice that although the number of possible 2D materials is huge, most of the research in this very competitive area is still focused on a reduced number of semiconducting and insulating materials (mainly graphene, $\mathrm{MoS}_{2}$ and hexagonal boron nitride (h-BN)). As a matter of fact, 2D materials exhibiting superconductivity or magnetism have been loosely investigated. Thus, only very 
recently superconductivity and magnetism in the $2 \mathrm{D}$ limit has also been put forward with the isolation and study of atomically-thin layers of metal dichalcogenides $\left(\mathrm{TaS}_{2}\right.$, for example), ${ }^{92}$ or transition metal halides $\left(\mathrm{CrI}_{3}\right)^{93}$, respectively. The main reason that explains this situation is the high chemical reactivity exhibited by these inorganic layers at ambient conditions. This seriously limits the isolation, manipulation and characterization of the 2D material, making necessary to work in a controlled atmosphere, free of oxygen and humidity. ${ }^{121}$

An alternative to overcome this limitation is to use a molecular approach. In this context Molecular Magnetism can offer a large variety of layered magnets based on coordination polymers, which are crystalline, chemically stable and easy to manipulate in open air. In the superconducting area the first coordination polymer exhibiting superconductivity with $\mathrm{T}_{\mathrm{c}}=0.25 \mathrm{~K}$ has also been recently discovered. ${ }^{94}$ Interestingly, this crystalline material shows a layered structure formed by a copper(II) benzenehexathiolate coordination polymer. Still, this molecular approach has some drawbacks: i) most of these coordination polymers are mechanically fragile and difficult to exfoliate down to the monolayer since often they are not formed by neutral layers weakly interacting through vdW forces, but by charged layers containing counter-ions in the inter-lamellar space and held together through electrostatic interactions. ii) A liquid-exfoliation procedure is the most common method used in chemistry to delaminate a layered coordination polymer. This wet method leads to small and surfacedefective nanosheets (with lateral sizes of the order of hundreds of nm, at best), which are often not exfoliated down to the monolayer and which are of insufficient quality to be used in 2D physics. Hence, a micromechanical physical procedure is mandatory to fulfil the high-quality requirement needed in 2D physics.

The first attempt to use a micromechanical (Scotch tape) procedure to exfoliate a layered coordination polymer was performed in the hybrid compounds $\left[\mathrm{Fe}^{\mathrm{III}}\left(\mathrm{acac}_{2}-\right.\right.$ trien) $]\left[\mathrm{Mn}^{\mathrm{II}} \mathrm{Cr}^{\mathrm{III}}\left(\mathrm{Cl}_{2} \mathrm{An}\right)_{3}\right] \cdot\left(\mathrm{CH}_{3} \mathrm{CN}\right)_{2} \quad$ and $\left[\mathrm{Fe}^{\mathrm{III}}\left(\mathrm{acac}_{2}-\right.\right.$ trien)][ $\left.\mathrm{Mn}^{\mathrm{II}} \mathrm{Cr}^{\mathrm{III}}\left(\mathrm{Br}_{2} \mathrm{An}\right)_{3}\right] \cdot\left(\mathrm{CH}_{3} \mathrm{CN}\right)_{2}$, which in bulk behave as ferrimagnets with a $\mathrm{T}_{\mathrm{c}}=$ $11 \mathrm{~K}$. In these materials the magnetic layer consists of a $2 \mathrm{D}$ anionic network formed by $\mathrm{Mn}(\mathrm{II})$ and $\mathrm{Cr}(\mathrm{III})$ ions linked through anilate (An) ligands (Figure 6 top). Interestingly, thanks to the pores created in this $2 \mathrm{D}$ framework, the counter-ions $\left[\mathrm{Fe}^{\mathrm{III}}\left(\mathrm{acac}_{2} \text {-trien) }\right]^{+}\right.$ are inserted inside it instead of being inserted in the interlamellar spacing. Thus, the resulting crystals may be viewed as formed by neutral hybrid layers assembled through 
vdW interactions. ${ }^{95}$ As for graphene, this feature facilitated the micromechanical exfoliation leading to the isolation of atomically-thin magnetic layers with thicknesses down to $1.5 \mathrm{~nm}$ and lateral sizes of the order of hundreds of nanometers. This is an interesting result, but still insufficient to perform a deep magnetic characterization in the 2D limit since the size of these layers is still too small (a lateral size of the order of tenths of microns is required). This limitation is closely related with the solution methods used for growing these crystals that, in contrast to what happens for purely inorganic materials, often afford very small crystals.

A second approach that avoids the use of solvents and leads to neutral layers has been provided by the family of coordination polymers formulated as $\left[\mathrm{Fe}(\operatorname{bimX})_{2}\right](\mathrm{HbimX}=$ derivative of benzimidazole, with $\mathrm{X}=\mathrm{H}, \mathrm{Cl}, \mathrm{Br}, \mathrm{CH}_{3}$, or $\left.\mathrm{NH}_{2}\right),{ }^{96}$ which behave as spincanted antiferromagnets with ordering temperatures, $\mathrm{T}_{\mathrm{N}}$, of $20 \mathrm{~K}$. From a chemical point of view, these compounds present several advantageous features: i) They are obtained as single crystals (typical size of $400 \mu \mathrm{m}$ ) by a dry chemical method in a sealed ampoule containing sublimable precursors. ii) These crystals contain neutral layers formed by distorted tetrahedral $\mathrm{Fe}^{\mathrm{II}}$ centers connected by benzimidazolate bridges (Figure 6 centre), which weakly interact with each other through van der Waals interactions established between the substituent groups $(\mathrm{X})$ of the benzimidazole ligand; therefore, they can be micromechanically exfoliated down to the monolayer, while preserving their crystallinity. Thus, ultrathin layers with lateral sizes of the order of tenths of microns, which are chemically stable in ambient conditions, can be easily isolated (Figure 6). iii) The pre-synthetic modification of the materials through the convenient choice of the substituent $\mathrm{X}$ gives rise to a surface tunability ranging, for example, from hydrophilic $\left(\mathrm{X}=\mathrm{NH}_{2}\right)$ to hydrophobic $(\mathrm{X}=\mathrm{H})$, albeit maintaining the inner physical property (i.e. magnetic order) in all the derivatives of the family. This result illustrates the ability of coordination chemistry for achieving a defect-free chemical functionalization of the surface of the 2D crystal, a feature that is impossible to achieve in graphene and other 2D inorganic materials, since a post-synthetic method has to be used for the functionalization; this inevitably leads to non homogeneous and highly defective surfaces that induce deep changes in the inner physical property of the 2D material. In the present case, this chemical functionalization allows to design chemically stable and mechanically robust 2D magnets, which may be integrated in spintronic devices. Thanks to this surface tunability, these functionalized layers may 
also be useful as ultrathin (and ultrasensitive) membranes for selective molecular sensing. ${ }^{96}$

To conclude this part one can assess that coordination chemistry can provide a suitable platform to chemically design and functionalize 2D molecule-based magnets. So far, only two examples of coordination polymers, which illustrate the isolation of robust and chemically stable magnetic layers of micrometric size, have been reported, although the complete characterization of the magnetism in the $2 \mathrm{D}$ limit remains a challenge. A bottom-up approach to grow these layers on surfaces should facilitate this goal as it will provide 2D magnets with larger lateral dimensions. On the other hand, we should note that these two materials are electrical insulators. Future directions should involve the search for 2D molecule-based magnets incorporating other properties of interest, such as electrical conductivity or spin-crossover. Initial attempts along these lines have started, ${ }^{97} 98$ although, for the moment, only the bulk properties have been studied in detail, owing to the small crystal sizes of these layered coordination frameworks and the difficulties encountered in their exfoliation. Finally, we should note that this topic is not limited to 2D molecule-based magnets. The possibility of combining magnetic molecules (or magnetic molecular materials) with inorganic 2D layers (semiconductors, conductors, superconductors or magnets) can afford the creation of molecular/2D heterostructures in which their properties can be tuned as a result of the coupling between the two nanomaterials. This concept has been illustrated in heterostructures formed by a layer of SCO nanoparticles deposited on graphene. One observes that the electrical properties of graphene change from a high conducting state to a low conducting one when the spin transition switch from low spin to high spin (Figure 6 bottom). ${ }^{99}$

\section{Outlook and perspectives}

In the past few years, Molecular Magnetism has shown an amazing advance towards the chemical design of novel magnetic molecules and materials, with an unprecedented control over their magnetic properties, even at the single-molecule level. In this article, I have shown how these advances have contributed to expand this field of research towards other emerging fields, such as Molecular Spintronics, Quantum Technologies, MOFs and 2D materials. This recent evolution has raised several issues in Molecular Magnetism which concern three interconnected aspects: The first is the chemical design 
and processing of novel magnetic molecules and molecule-based materials, the second involves the understanding of the properties of these molecular systems, and the third the use of this newly gained knowledge to develop functional nanostructures and applications. A list with some potential research topics in these three aspects is summarized in Table 1 for each one of the four crossing fields. An inspection of this table allows us to make some remarks:

1) In the context of the Molecular Magnetism, these crossing fields are not independent but converge towards some common challenges. For example, molecular spintronics and quantum technologies share common interests for the SMMs, while 2D magnetic MOFs impact not only in the fields of magnetic MOFs and 2D magnetic materials, but also in molecular spintronics.

2) This recent evolution of Molecular Magnetism towards nanomagnetism and nanomaterials, with an increasing tendency towards the applications, has been triggered by the general evolution experienced in materials science towards nanoscience and nanotechnology. However, most of the current research topics are still focused on basic science.

3) As far as the materials are concerned, the potential research topics emphasize the increasing interest for the design of hybrid molecular/inorganic structures and interfaces exhibiting new properties thanks to the coupling between these two components. This will provide new opportunities such as that of tuning the properties of the material or the interface through external stimuli, including temperature, light, strain or gating. This includes the design of molecular/2D heterostructures in which the interaction of the magnetic molecular component with the 2D material can control its electrical, optical or magnetic properties. In addition, Molecular Magnetism has always been characterized for the search of new materials exhibiting magnetic phenomena of interest. Thus, it is expected that the molecular analogues of some of the materials that are currently revolutionizing the magnetism will emerge in the near future. Besides 2D magnets, I can mention for example molecule-based magnets hosting or interacting with skyrmions, ${ }^{100}$ molecular-based quantum spin liquids, ${ }^{101}$ molecular magnonic materials and devices ${ }^{102}$ or hybrid heterostructures exhibiting quantum phases. ${ }^{103}$ 
4) In all the discussion, I have chosen the crossroads that are close to physics and materials chemistry, as they are the ones that are currently focusing most of the attention. Still, the inspiration from biology and its intersection with Molecular Magnetism should not be neglected in the future, even more when we observe that the biological aspects and the medical applications are starting to develop in neighbouring areas such as molecular electronics ${ }^{104}$ and MOFs. ${ }^{105}$ 
Table 1. List of potential research topics in each research crossroad

\begin{tabular}{|c|c|c|c|}
\hline & Molecules \& Materials & Fundamental issues & Applications \\
\hline $\begin{array}{l}\text { Molecular } \\
\text { Spintronics }\end{array}$ & $\begin{array}{l}\text { - Design of robust magnetic } \\
\text { molecules based on } \\
\text { coordination chemistry } \\
\text { (SMMs \& SCO) for their } \\
\text { integration in spintronic } \\
\text { structures. } \\
\text { - Molecular/inorganic } \\
\text { hybrid spin interfaces based } \\
\text { on functional molecules } \\
\text { (chiral molecules, } \\
\text { luminescent organic } \\
\text { radicals,...). } \\
\text { •Molecular/AF hybrid } \\
\text { interfaces. }\end{array}$ & $\begin{array}{l}\text { - Active control of the } \\
\text { hybrid spin interfaces. } \\
\text { - Understanding spin } \\
\text { injection / transport in } \\
\text { molecular materials. } \\
\text { - Understanding the spin } \\
\text { transition at the interface. }\end{array}$ & $\begin{array}{l}\text { - Multifunctional } \\
\text { spintronic devices (spin- } \\
\text { OLEDs, spin-OFETs, } \\
\text { spin-molecular } \\
\text { photovoltaics). } \\
\text { - New concepts in } \\
\text { molecular spintronics } \\
\text { (smart spin valves, } \\
\text { skyrmion-based spintronic } \\
\text { devices, hybrid magnonic } \\
\text { devices,...). } \\
\text { - Single-molecule } \\
\text { spintronic nanodevices. }\end{array}$ \\
\hline $\begin{array}{l}\text { Molecular } \\
\text { Spins in } \\
\text { Quantum } \\
\text { Technologies }\end{array}$ & $\begin{array}{l}\text { - Design of molecular spin } \\
\text { qubits exhibiting large } \\
\text { coherence times. } \\
\text { - Design of molecules } \\
\text { containing several } \\
\text { addressable spin qubits } \\
\text { (qudit molecules). }\end{array}$ & $\begin{array}{l}\text { - Control of the spin- } \\
\text { photon coupling in } \\
\text { molecular spin qubits. } \\
\text { - Role of molecular } \\
\text { vibrations and phonons on } \\
\text { the quantum coherence. } \\
\text { - Interactions of molecular } \\
\text { spin qubits with } \\
\text { superconductors }\end{array}$ & $\begin{array}{l}\text { - Quantum processors } \\
\text { based on single magnetic } \\
\text { molecules. } \\
\text { - Implementing scalable } \\
\text { architectures coupling } \\
\text { molecular spin qubits to } \\
\text { photons in a } \\
\text { superconducting } \\
\text { resonator. }\end{array}$ \\
\hline $\begin{array}{l}\text { Magnetic } \\
\text { MOFs }\end{array}$ & $\begin{array}{l}\text {-2D magnetic MOFs. } \\
\text { - Conductive magnetic } \\
\text { MOFs. } \\
\text { - Magnetic MOFs for } \\
\text { quantum information. }\end{array}$ & $\begin{array}{l}\text { - Interplay between } \\
\text { conductivity and } \\
\text { magnetism in conductive } \\
\text { magnetic MOFs. } \\
\text { - Interplay between } \\
\text { molecular bistability and } \\
\text { magnetism in magnetic } \\
\text { MOFs. }\end{array}$ & $\begin{array}{l}\text { - Selective chemosensors. } \\
\text { - Processing MOFs as } \\
\text { ultrathin films or } \\
\text { nanoparticles for } \\
\text { integration in hybrid } \\
\text { nanostructures }\end{array}$ \\
\hline $\begin{array}{l}\text { 2D Magnetic } \\
\text { Materials }\end{array}$ & $\begin{array}{l}\text { - Growth of 2D magnetic } \\
\text { MOFs on surfaces. } \\
\text { - 2D spin crossover MOFs. } \\
\text { - Hybrid heterostructures } \\
\text { interfacing 2D magnetic } \\
\text { MOFs with other functional } \\
\text { components (molecules, 2D } \\
\text { materials, 3D substrates). } \\
\text { - Chemical functionalization } \\
\text { of 2D magnetic MOFs. } \\
\text { - Nanocomposites } \\
\text { containing 2D magnetic } \\
\text { MOFs. } \\
\text { - Hybrid heterostructures } \\
\text { interfacing inorganic 2D } \\
\text { materials with magnetic } \\
\text { molecular layers (magnetic } \\
\text { molecules, magnetic } \\
\text { nanoparticles, magnetic } \\
\text { MOFs). }\end{array}$ & $\begin{array}{l}\text { - Magnetic measurements } \\
\text { in individual sheets of 2D } \\
\text { molecule-based magnets. } \\
\text { - Physical characterization } \\
\text { of 2D molecule-based } \\
\text { antiferromagnets. } \\
\text { - Proximity effects in } \\
\text { hybrid heterostructures } \\
\text { containing 2D magnetic } \\
\text { MOFs. } \\
\text { - Hybridization of } \\
\text { molecules with 2D } \\
\text { magnetic MOFs. } \\
\text { - Interaction of molecular } \\
\text { spin qubits with 2D } \\
\text { superconductors. } \\
\text { - Tuning the properties of } \\
\text { inorganic 2D materials via } \\
\text { their coupling with } \\
\text { magnetic molecular } \\
\text { systems. }\end{array}$ & $\begin{array}{l}\text { - Spintronic devices } \\
\text { containing 2D magnetic } \\
\text { MOFs. } \\
\text { - Magnonic devices based } \\
\text { on 2D molecule-based } \\
\text { antiferromagnets. } \\
\text { - Ultrathin devices based } \\
\text { on molecular/2D } \\
\text { heterostructures. } \\
\text { - Membranes based on } \\
\text { functionalized 2D MOFs. }\end{array}$ \\
\hline
\end{tabular}




\section{Box 1| Basic of spintronics}

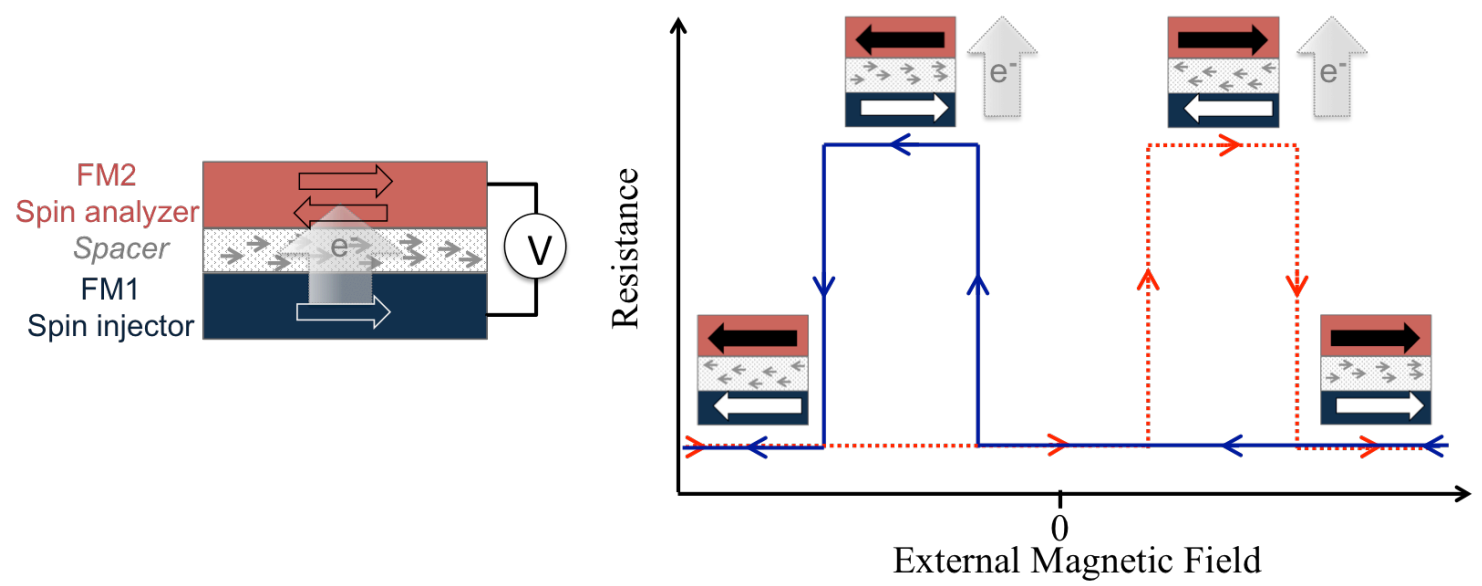

A basic spintronic device consists of a thin layer of a non-magnetic material (spacer) sandwiched between two ferromagnetic layers (FM1 and FM2) working as electrodes. ${ }^{106,107}$ One of these electrodes - usually the hardest one- plays the role of spin injector, injecting a spin polarized current into the spacer, while the second oneusually the softest one - acts as spin detector, or spin analyzer (see left figure). This trilayer heterostructure is referred to as "spin valve" (SV) or "magnetic tunnel junction" (MTJ) when the spacer is (semi)conducting or insulator, respectively. In these devices a spin-dependent electron transport is observed, with their resistance values depending on the relative orientation of the magnetization of the FM electrodes. For the antiparallel alignment, both spin-up and spin-down electrons suffer many scattering processes in someone of the FM layers, resulting in a high resistance configuration. For the parallel arrangement, the spin polarized electrons travel nearly unscattered from the spin injector to the spin detector, which results in a low resistance configuration. In the MTJs the insulating layer is so thin that electrons tunnel through the insulating barrier, preserving their spin, when a bias voltage is applied between the two metallic electrodes.

In order to measure these changes in the resistance an external magnetic field is applied, which is able to switch the relative orientation of the magnetizations of the two FM electrodes, owing to their different coercive fields. So, a strong enough external magnetic field parallel to the electrodes will force them to point their magnetization in the same orientation, leading to a low resistance configuration $\left(R_{P}\right)$. By changing the orientation of the external magnetic field, the electrode with the smaller coercive field 
will change its magnetization first, giving rise to opposite orientations of the magnetization directions of both electrodes, leading to a high resistance configuration $\left(\mathrm{R}_{\mathrm{AP}}\right)$. Thus, the magnetoresistance $(\mathrm{MR})$ that characterizes the device will be defined as $\mathrm{MR}=\left(\mathrm{R}_{\mathrm{P}}-\mathrm{R}_{\mathrm{AP}}\right) / \mathrm{R}_{\mathrm{P}}$ and will exhibit two different states. These magnetoresistive effects are called giant magnetoresistance (GMR) and tunneling magnetoresistance, (TMR) for SVs and MTJs, respectively. A schematic representation of an ideal resistance measurement $v s$. an external magnetic field is shown in the right image. 


\section{Box 2| Concepts in quantum computation with spin qubits}
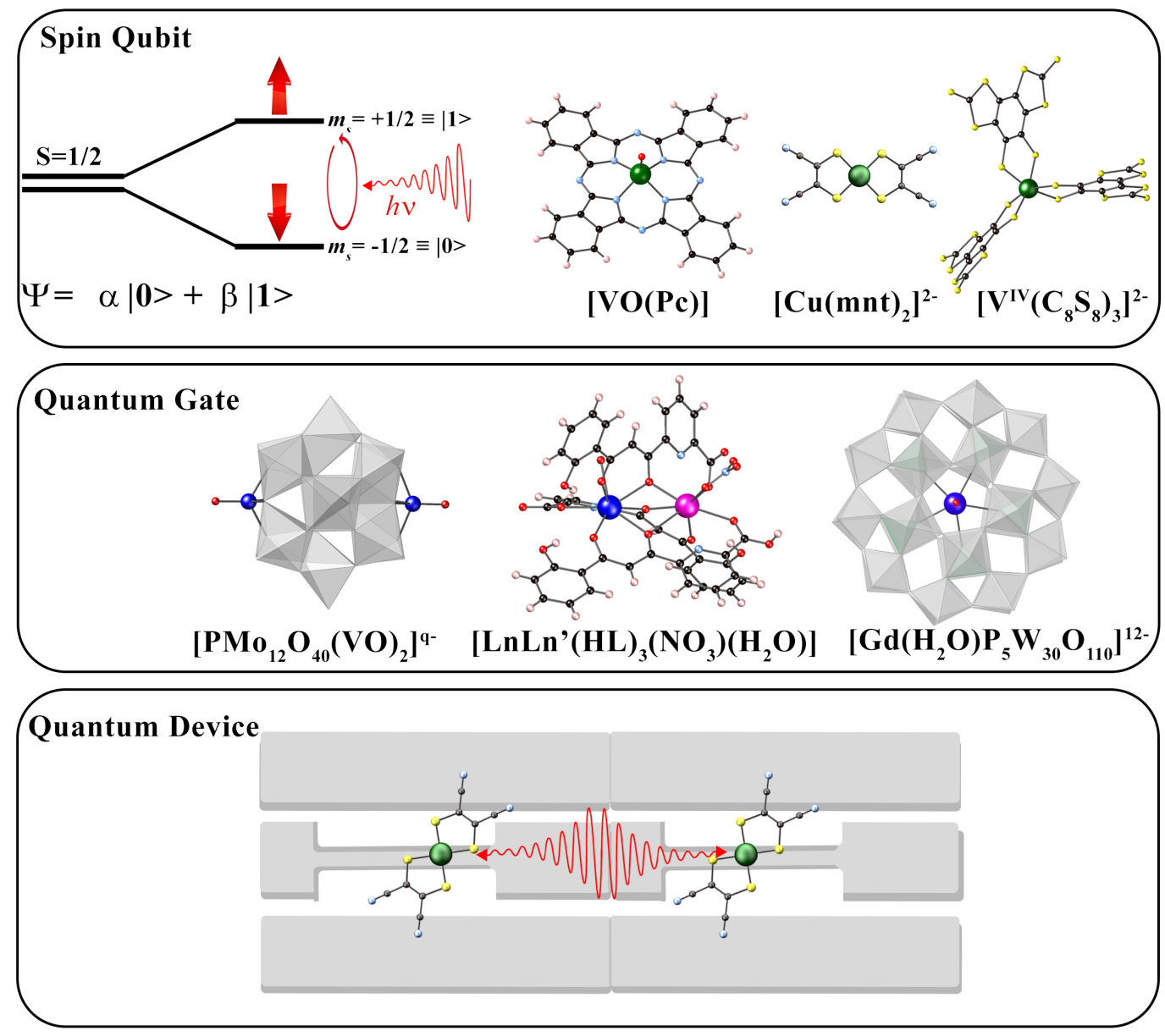

The research in the area of quantum computation is a three step process that goes from the design of robust quantum bits (qubits), to the coupling between them for implementing quantum logic gates, and, finally, to the integration of these qubits into scalable devices to store and process the quantum information. In the figure molecular examples that illustrate each one of these steps are displayed.

Qubits. A quantum bit or "qubit" is a quantum system with two eigenstates $\mid 0>$ and $\mid 1>$. The state of a qubit can be expressed as a quantum superposition of these two states: $|\Psi>=\alpha| 0>+\beta \mid 1>$. To be usable in practice, we must also be able to create, manipulate and readout the qubit states. The concept of qubit can be generalized to multi-level quantum systems giving rise to qudits, where $d$ is the number of accessible quantum states. The current sources of qubits include superconducting circuits, semiconductor quantum dots, atoms or ions in optical traps or photons in resonant cavities. Spin states (electronic and/or nuclear) of atoms or molecules are also a source of qubits, since spins 
are naturally quantum objects. For example, $S=1 / 2$ is a doublet with $m_{S}= \pm 1 / 2$ that splits in two distinguishable levels under the influence of an external magnetic field (Zeeman effect). The two most challenging requirements for qubits are coherence and scalability. The former is quantified by the phase memory time $T_{2}$ (the loss of quantum information by interaction with the environment is known as decoherence) and for electronic spins can be estimated from pulsed EPR measurements. The later is defined as a systematic approach to increase the number of qubits, while maintaining the ability to create, manipulate and read-out their states and preserve their coherence). In this context, each one of the available approaches to get qubits presents advantages and disadvantages. Photons, for example, are extremely robust against decoherence, but it is very difficult to effect arbitrary manipulations of quantum states involving more than a single photon. The current consensus in the field is that no short-term solution based on current technology is able to scale up to large numbers of coherent qubits.

Focusing on spin qubits, magnetic impurities in solids, like nitrogen-vacancy (NV) colour centres in diamond, phosphorus impurities in silicon, or simple inorganic salts doped with lanthanide ions, are the most popular ones. For both, NV centers and phosphorus in silicon, long-distance qubit couplings have been achieved, ${ }^{108,109}$ although scalability is not yet accessible. In special situations lanthanide ions as dopants have reached record coherence times with $T_{2}$ values as high as 6 hours at $2 \mathrm{~K}$ in optimized conditions, measured in bulk samples. ${ }^{110}$ One of the main sources of decoherence in solid state spin qubits, especially at low temperature, is the magnetic noise, which includes dipolar and hyperfine interactions with all electronic and nuclear spins in the sample or in the device. These interactions involve an uncontrolled dispersion of the spin information throughout the sample. Additionally, as the temperature rises, so does the effect of vibrations, whether local or extended (phonons). These allow an energy exchange between the spin system and the thermal bath, thus offering a pathway for the magnetic relaxation and a complete loss of the spin information.

Quantum gates. The application of a logical operation to one or more qubits is known as a quantum gate or qugate. For example, the qugate "Controlled-NOT" (or CNOT) acting on a pair of spin qubits, $\mathrm{A}$ and $\mathrm{B}$, flips the orientation of the spin qubit $\mathrm{B}$, and vice versa if, and only if, the spin qubit A is pointing "up": CNOT $|10>=| 11>$. Similarly, the "SWAP" gate acts on a pair of spin qubits A and B, exchanging their values. Finally, variations on these gates are possible, for example the "sqrt(SWAP)" creates an 
entangled state such that, if the gate is applied twice in a row, the values of the qubits are exchanged: $\operatorname{sqrt}(\mathrm{SWAP}) * \operatorname{sqrt}(\mathrm{SWAP})|01>=\mathrm{SWAP}| 01>=\mid 10>$.

Quantum devices. The final step in quantum computation involves the processing of quantum information. This requires the use of quantum devices that allow to scale up the number of qubits coherently coupled. One promising approach to reach this goal is based on the use of superconducting resonators that act as microwave transmission lines. In these resonators an individual microwave photon can couple any pair of spin qubits (no matter their mutual distance). Recent results in this topic include measuring the quantum limit of spin relaxation in nitrogen-vacancy centers coupled to resonating microwaves and finding that it is limited by phonon vacuum modes (that is, by vibrations) even at temperatures in the $50-200 \mathrm{mK}$ range. ${ }^{11}$ 


\section{Box 3| Functional MOFs}

Metal-organic frameworks (MOFs) are open crystalline frameworks with permanent porosity that are constructed from the assembly of inorganic sub-units (metal centres, clusters, chains...) and organic linkers (carboxylates, phosphonates, azolates, etc.). Their high versatility is a result of the adaptable nature of the organic ligands and their variety of coordination modes, combined with the geometry of the constitutive metal ions, which provides an assortment of topologies that can be intended by chemical design. ${ }^{12}$

A current trend in this field is that of designing MOFs with electronic functionalities (showing electrical, magnetic or optical properties). The different electronic functionalities that can be implemented in these porous solids can arise in three different manners: (i) by implementation of the desired functionality in the framework itself; (ii) by insertion of functional molecules in the pores of the MOF; (iii) by a synergistic effect through combination of the previous two components (see figure below).

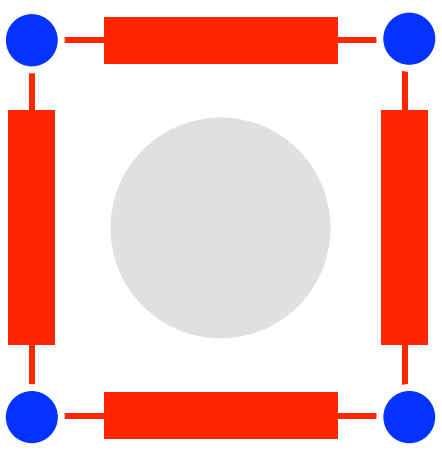

(i)

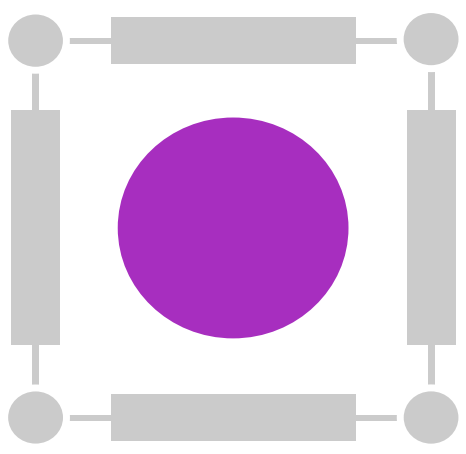

(ii)

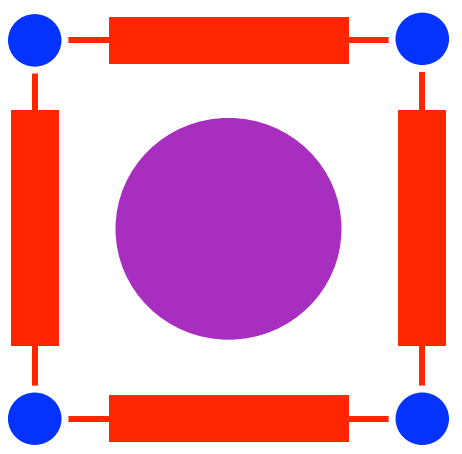

(iii)

Functional framework

Functional framework

\&

Functional guest 


\section{Box 4| 2D materials: Complexity vs. quality of the material and fields of application}

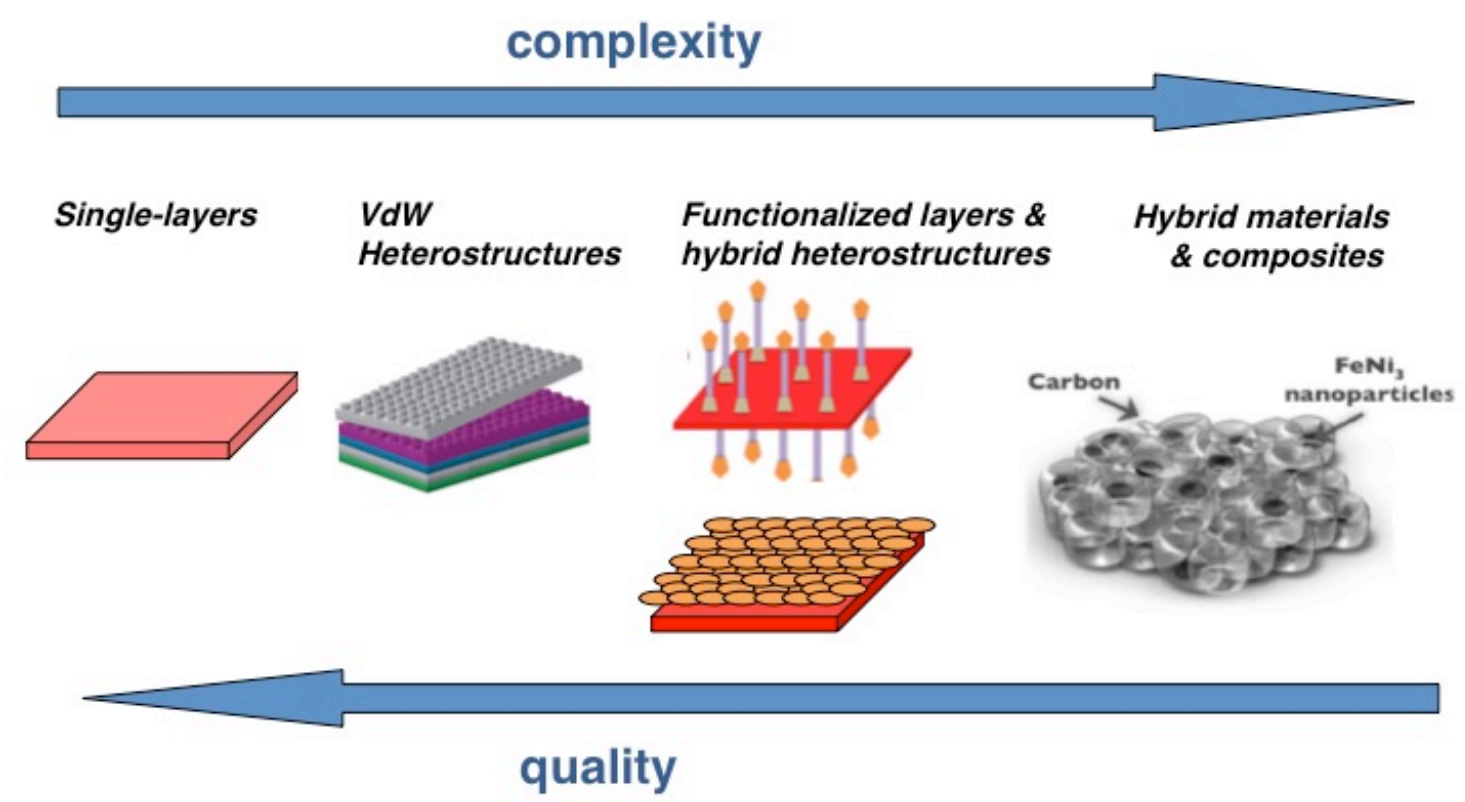

Since the isolation of graphene in 2004, there has been an explosion in the search for other atomically thin layers (aka. 2D materials). ${ }^{113,114,115}$ The majority of these systems are layered materials that in bulk show stacked structures with weak van der Waals (vdW) interactions between adjacent sheets but with strong covalent bonding within each of the layers, thus permitting their mechanical or chemical exfoliation in ultrathin 2D crystals. ${ }^{116}$ These $2 \mathrm{D}$ materials cover the entire range of electronic and magnetic properties and chemical compositions: from insulators to superconductors, from diamagnetic to ferromagnetic (FM) and from metallic to non-metallic compositions. These $2 \mathrm{D}$ materials can be integrated in more complex structures when entering in contact with other materials of different dimensionalities. Thus, when they are assembled with other 2D materials they afford vdW heterostructures in which different monolayers are held together by vdW forces. ${ }^{117}$ The next degree of complexity is to chemically functionalize the $2 \mathrm{D}$ material by anchoring molecular species or other chemical nano-objects on its surface via vdW forces, electrostatic interactions or chemical bonds. ${ }^{118}$ This includes hybrid molecular/2D heterostructures, formed by 
placing molecular species on the top of a $2 \mathrm{D}$ material, as well as mixed vdW 1D/2D and 2D/3D heterostructures. In a final step, one can imagine the formation of hybrid materials and nanocomposites from the combination of $2 \mathrm{D}$ materials with other nanomaterials. All these possibilities are schematized in the figure. These have opened new avenues for both studying fundamental physical phenomena and developing applied research towards the design of novel devices. ${ }^{119,120}$ In fact, nowadays the study of $2 \mathrm{D}$ materials and vdW heterostructures is a hot topic in physics due to the specific physical properties that can appear in the $2 \mathrm{D}$ limit, ${ }^{121}$ and to the use of these materials as components in electronic devices in a new field known as graphene-based electronics (or, in general, 2D electronics). ${ }^{122,123}$ On the other hand, these materials have also shown to be of interest in materials science since they can provide a source of advanced materials in a field known as graphene-based composites. ${ }^{124}$ Clearly, the requirement of the 2D material will be different depending on the applications (see Figure). Thus, 2D physics and $2 \mathrm{D}$ electronics are highly demanding with respect to the quality of the $2 \mathrm{D}$ materials (they require low defect density 2D crystals and employ all-dry fabrication techniques for their isolation). In contrast, the 2D composite applications can tolerate lower quality of the materials, which is often compatible with the solution techniques developed in chemistry to prepare functionalized 2D materials and hybrid materials. 


\section{REFERENCES}

${ }^{1}$ Willett, R. D., Gatteschi, D. \& Kahn, O. Magneto-structural correlations in exchange coupled systems. NATO-ASI Series. D. Reidel Pub. Co. 1985.

${ }^{2}$ Kahn, O. Dinuclear complexes with predictable magnetic properties. Angew. Chem. Int. Ed. 24, 834-850 (1985).

${ }^{3}$ Drillon, M., Coronado, E., Beltrán, D. \& Georges, R. Classical treatment of a

Heisenberg linear chain with spin alternation; application to the $\mathrm{MnNi}(\mathrm{EDTA}) \cdot 6\left(\mathrm{H}_{2} \mathrm{O}\right)$. Chem. Phys. 79, 449-453 (1983).

${ }^{4}$ Kahn, O. Molecular Magnetism VCH 1993.

${ }^{5}$ Miyasaka, H., Julve, M., Yamashita, M. \& Clerac, R. Slow dynamics of the magnetization in one-dimensional coordination polymers: single-chain magnets. Inorg. Chem. 48, 3420-3437 (2009).

${ }^{6}$ Sessoli, R. et al. Strong magneto-chiral dichroism in a paramagnetic molecular helix observed by hard X-rays. Nature Phys. 11, 69-74 (2015).

${ }^{7}$ Miller, J. S. Organic- and molecule-based magnets. Mater. Today 17, 224-235 (2014).

${ }^{8}$ Caneschi, A., Gatteschi, D., Sessoli, R. \& Rey, P. Toward molecular magnets- the metal-radical approach. Acc. Chem. Res. 22, 392-398 (1989).

${ }^{9}$ Stumpf, H. O., Ouahab, L., Pei, Y., Grandjean, d. \& Kahn, O. A molecular-based magnet with a fully interlocked 3-dimensional structure. Science 261, 447-449 (1993).

${ }^{10}$ Tamaki, H. et al. Design of metal-complex magnets- syntheses and magnetic properties of mixed-metal assemblies $\left\{\mathrm{NBu}_{4}\left[\mathrm{MCr}(\mathrm{ox})_{3}\right]\right\}_{\mathrm{x}}\left(\mathrm{NBu}_{4}{ }^{+}=\operatorname{tetra}(n\right.$-butyl $)$ ammonium ion, $\mathrm{ox}^{2-}=$ oxalate ion, $\left.\mathrm{M}=\mathrm{Mn}^{2+}, \mathrm{Fe}^{2+}, \mathrm{Co}^{2+}, \mathrm{Ni}^{2+}, \mathrm{Cu}^{2+}, \mathrm{Zn}^{2+}\right)$. J. Am. Chem. Soc. 114, 6974-6979 (1992).

${ }^{11}$ Ferlay, S. Mallah, T., Ouahès, R., Veillet, P. \& Verdaguer, M. A room-temperature organometallic magnet based on Prussian blue. Nature 378, 701-703 (1995).

${ }^{12}$ Gütlich, P., Hauser, A. \& Spiering, H. Thermal and optical switching of Iron(II) complexes. Angew. Chem. Int. Ed. 33, 2024-2054 (1994).

${ }^{13}$ Real, J.A., Gaspar, A.B., Muñoz, M.C. Thermal, pressure and light switchable spincrossover materials. Dalton Trans. 12, 2062-2079 (2005).

${ }^{14}$ Linares, J., Codjovi, E. \& Garcia, Y. Pressure and temperature spin crossover sensors with optical detection. Sensors 12, 4479-4492 (2012).

${ }^{15}$ Molnár, G., Rat, S., Salmon, L., Nicolazzi, W. \& Bousseksou, A. Adv. Mater. 30, 1703862 (2018).

${ }^{16}$ Kahn, O. \& Jay Martínez, C. Spin-transition polymers: From molecular materials toward memory devices. Science 279, 44-48 (1998).

${ }^{17}$ Gatteschi, D. Molecular Magnetism: A basis for new materials. Adv. Mat. 6, 635-645 (1994).

${ }^{18}$ Sessoli, R., Gatteschi, D., Caneschi, A. \& Novak, M. A. Magnetic bistability in a metal-ion cluster. Nature 365, 141-143 (1993).

${ }^{19}$ Thomas, L. et al. Macroscopic quantum tunnelling of magnetization in a single crystal of nanomagnets. Nature 383, 145-147 (1996).

${ }^{20}$ Friedman, J. R., Sarachik, M. P., Tejada, J. \& Ziolo, R. Macroscopic measurement of resonant magnetization tunneling in high-spin molecules. Phys. Rev. Lett. 76, 38303833 (1996).

${ }^{21} \mathrm{https}: / /$ www.nature.com/milestones/milespin/full/milespin22.html

${ }^{22}$ Coronado, E. \& Day, P. Magnetic molecular conductors. Chem. Rev. 104, 5419-5448 (2004). 
${ }^{23}$ Coronado, E., Galán-Mascarós, J.R., Gómez-García, C. J. \& Laukhin, V. Nature 408, 447-449 (2000).

${ }^{24}$ Coronado, E. Gómez-García, C. J., Nuez, A., Romero, F. M. \& Waerenborgh, J. C. Synthesis, chirality and magnetic properties of bimetallic cyanide-bridged twodimensional ferromagnets. Chem. Mater. 18, 2670-2681 (2006).

${ }^{25}$ Train, C. et al. Strong magneto-chiral dichroism in enantiopure chiral ferromangets. Nature Mater. 7, 729-734 (2008).

${ }^{26}$ Maspoch, D. et al. A nanoporous molecular magnet with reversible solvent-induced mechanical and magnetic properties. Nature Mater. 2, 190-195 (2003).

${ }^{27}$ Sato, O., Iyoda, T., Fujishima, A. \& Hashimoto, K. Photoinduced magnetization of a cobalt-iron cyanide. Science 272, 704-705 (1996).

${ }^{28}$ Coronado, E. et al. Pressure-tuning of magnetism and linkage isomerism in iron(II) hexacyanochromate. J. Am. Chem. Soc. 127, 4580-4581 (2005).

${ }^{29}$ Ohkoshi, S. et al. 90-degree optical switching of output second-harmonic light in chiral photomagnet. Nature Photonics 8, 65-71 (2014).

${ }^{30}$ Abellán, G., Martí-Gastaldo, C., Ribera, A. \& Coronado, E. Hybrid materials based on magnetic layered double hydroxides: A molecular perspective. Acc. Chem. Res. 48, 1601-1611 (2015).

${ }^{31}$ Bogani, L. \& Wernsdorfer, W. Molecular spintronics using single-molecule magnets. Nature Mater. 7, 179-186 (2008).

${ }^{32}$ Camarero, J. \& Coronado, E. Molecular vs. Inorganic spintronics: The role of molecular materials and single molecules. J. Mater. Chem., 19, 1678-1684 (2009).

${ }^{33}$ Sanvito, S. Molecular spintronics. Chem. Soc. Rev. 40, 3336-3355 (2011).

${ }^{34}$ Dediu, V., Murgia, M., Matacotta, M. C., Taliani, C. \& Barbanera, S. Room temperature spin polarized injection in organic semiconductor. Solid State Commun. 122, 181-184 (2002).

${ }^{35}$ Xiong, Z. H., Wu, D., Vardeny, Z. V. \& Shi, J. Giant magnetoresistance in organic spin-valves. Nature 427, 821-824 (2004).

${ }^{36}$ Dediu, V.A., Hueso, L. E., Bergenti, I. \& Taliani, C. Spin routes in organic semiconductors. Nature Mater. 8, 707 (2009).

${ }^{37}$ Nguyen, T. D., Ehrenfreund, E. \&Vardeny, Z. V. Spin-polarized light emitting diode based on an organic bipolar spin valve. Science 337, 204 (2012).

${ }^{38}$ Prieto, J. P. et al. Enhancing light Emission in interface Engineered Spin-OLEDs through spin-polarized injection at high voltages. Adv. Mater. 31, 1806817 (2019).

${ }^{39}$ Sun, X. et al. A molecular spin-photovoltaic device. Science 357, 677 (2017).

${ }^{40}$ Mannini, M. et al. Quantum tunnelling of the magnetization in a monolayer of oriented single-molecule magnets. Nature 468, 417-421 (2010).

${ }^{41}$ C. Wäckerlin, C. et al. Giant hysteresis of single-molecule magnets adsorbed on a non-magnetic insulator. Adv. Mater. 28, 5195-5199 (2016).

${ }^{42}$ Martínez, R. V. et al. Nanoscale deposition of single-molecule magnets onto $\mathrm{SiO}_{2}$ patterns. Adv. Mater. 19, 291-295 (2007).

${ }^{43}$ Atodiresei, N. et al. Design of the local spin polarization at the organic-ferromagnetic interface Phys. Rev. Lett. 105, 066601 (2010).

${ }^{44}$ Aragonés, A. C. et al. Large conductance switching in a single-molecule device through room temperature spin-dependent transport. Nano Lett. 16, 218-226 (2016).

${ }^{45}$ Bedoya-Pinto, A, Prima-García, H., Casanova, F., Coronado, E. \& Hueso, L. E. Spinpolarized hopping transport in magnetically tunable rare-earth quinolines. $A d v$.

Electron. Mater. 1, 1500065 (2015).

${ }^{46}$ Poggini, L. et al. An organic spin valve embedding a self assembled monolayer of organic radicals. Adv. Mater. Interfaces 3, 1500855 (2016). 
${ }^{47}$ Cucinotta, G. et al. Tuning of a vertical spin valve with a monolayer of single molecule magnets. Adv. Funct. Mater. 50, 1703600 (2017).

${ }^{48}$ Miralles, S. G. et al. Sublimable chloroquinolinate lanthanoid single-ion magnets deposited on ferromagnetic electrodes. Chem. Sci. 9, 199-208 (2018).

${ }^{49}$ Bedoya-Pinto, A. et al. Interface-assisted sign inversion of the magnetoresistance in spin valves based on novel lanthanide quinoline molecules. Adv. Funct. Mater. 28, 1702099 (2017).

${ }^{50}$ Sanvito, S. The rise of spinterface science. Nature Phys. 6, 562-564 (2010).

${ }^{51}$ Cinchetti, M. Dediu, A. \& Hueso, L.E. Activating the molecular spinterface. Nature Mater. 16, 507 (2017).

${ }^{52}$ Forment-Aliaga, A. \& Coronado, E. Hybrid interfaces in molecular spintronics. Chem. Record 18, 737-748 (2018).

${ }^{53}$ Prins, F., Monrabal-capilla, M., Osorio, E. A., Coronado, E. \& van der Zant, H. S. J. Room-temperature electrical addressing of a bistable spin-crossover molecular system. Adv. Mater. 23, 1545-1549 (2011).

${ }^{54}$ Dugay, J. et al. Spin switching in electronic devices based on 2D assemblies of spincrossover nanoparticles. Adv. Mater. 27, 1288-1293 (2015).

55 Torres-Cavanillas, R. et al. Design of bistable gold@spin-crossover core-shell nanoparticles showing large electrical responses for the spin switching. Adv. Mater., DOI: 10.1002/adma.201900039.

${ }^{56}$ Holovchenko, A. et al. Near Room-Temperature Memory Devices Based on Hybrid Spin-Crossover@SiO $\mathrm{Sin}_{2}$ Nanoparticles Coupled to Single-Layer Graphene Nanoelectrodes. Adv. Mater. 28, 7228-7233 (2016).

${ }^{57}$ Vincent, R., Klyatskaya, S., Ruben, M., Wernsdorfer, W. \& Balestro, F. Electronic read-out of a single nuclear spin using a molecular spin transistor. Nature 488, 357-360 (2012).

${ }^{58}$ Thiele, S. et al. Electrically driven nuclear spin resonance in single-molecule magnets. Science 344, 1135-1138 (2014).

${ }^{59}$ Moreno-Pineda, E., Godfrin, C., Balestro, F., Wernsdorfer, W. \& Ruben, M.

Molecular spin qudits for quantum algorithms. Chem. Soc. Rev. 47, 501-513 (2018).

${ }^{60}$ Clemente-Juan, J. M., Coronado, E. \& Gaita-Ariño, A. Chem. Soc. Rev. 41, 74647478 (2012)

${ }^{61}$ Gaita-Ariño, A., Luis, F., Hill, S. \& Coronado, E. Molecular Spins for quantum computation. Nature Chem. 11, 301-309 (2019)

${ }^{62}$ Escalera-Moreno, L., Baldoví, J.J., Gaita-Ariño, A. \& Coronado, E. Spin states, vibrations and spin relaxation in molecular nanomagnets and spin qubits: a critical perspective. Chem. Sci. 9, 3265-3275 (2018).

${ }^{63}$ Zadrozny, J. M., Niklas, J., Poluektov, O. G. \& Freedman, D. E. ACS Cent. Sci. 1, 488-492 (2015).

${ }^{64}$ Bader, K. et al. Room temperature quantum coherence in a potential molecular qubit. Nature Commun. 5, 5304 (2014).

${ }^{65}$ Atzori, M. et al. Room-Temperature Quantum Coherence and Rabi Oscillations in Vanadyl Phthalocyanine: Toward Multifunctional Molecular Spin Qubits. J. Am. Chem. Soc. 138, 2154-2157 (2016).

${ }^{66}$ Shiddiq M. et al. Enhancing coherence in molecular spin qubits via atomic clock transitions. Nature 531, 348-351 (2016).

${ }^{67}$ Lehmann, J., Gaita-Arin̄o, A., Coronado, E. \& Loss, D. Spin qubits with electrically gated polyoxometalate molecules. Nature Nanotech. 2, 312-317 (2007). 
${ }^{68}$ Ferrando-Soria, J. et al. A modular design of molecular qubits to implement molecular quantum gates. Nature Commun. 7, 11377 (2016).

${ }^{69}$ Aromi, G., Aguilá, D., Gamez, P., Luis, F. \& Roubeau, O. Design of magnetic coordination complexes for quantum computing. Chem. Soc. Rev. 41, 537-546 (2012)

${ }^{70}$ Jenkins, M. D. et al. Coherent manipulation of three-qubit states in a molecular single-ion magnet. Phys. Rev. B 95, 064423 (2017).

${ }^{71}$ Jenkins, M. D. et al. Coupling single-molecule magnets to quantum circuits. New J. Phys. 15, 095007 (2013).

${ }^{72}$ Wang, C., Liu, D. \& Lin, W. Metal-organic Frameworks as A Tunable Platform for Designing Functional Molecular Materials. J. Am. Chem. Soc. 135, 13222-13234 (2013)

${ }^{73}$ Sun, L., Campbell, M. G. \& Dinca, M. Electrically conductive porous metal-organic frameworks. Angew. Chem. Int. Ed. 55, 3566-3579 (2016).

${ }^{74}$ Park, J. G. et al. Long Charge Delocalization and Bulk Electronic Conductivity in the Mixed-Valence Metal-Organic Framework Fe(1,2,3-triazolate $)_{2}\left(\mathrm{BF}_{4}\right)_{\mathrm{x}} . \mathrm{J}$. Am. Chem.

Soc. 140, 8526-8534 (2018).

${ }^{75}$ Xie, L. S. et al. Tunable Mixed-Valence Doping toward Record Electrical Conductivity in a Three-Dimensional Metal-Organic Framework J. Am. Chem. Soc.

140, 7411-7414 (2018).

${ }^{76}$ Aubrey, M. L. et al. Nature Mater. 17, 625-632 (2018).

${ }^{77}$ Z.Hu, Z., Deibert, B. J. \& Li, J. Luminescent metal-organic frameworks for chemical sensing and explosive detection. Chem. Soc. Rev. 43, 5815-5840 (2014).

${ }^{78} \mathrm{Xu}, \mathrm{L} .-\mathrm{J} ., \mathrm{Xu}, \mathrm{G} .-\mathrm{T}$., Chen, Z.-N. Recent advances in lanthanide luminiscence with metal-organic chromophores as sensitizers. Coord. Chem. Rev. 273-274, 47-62 (2014). ${ }^{79}$ Coronado, E. \& Mínguez Espallargas, G. Dynamic Magnetic MOFs. Chem. Soc. Rev. 42, 1525 (2013).

${ }^{80}$ Mínguez Espallargas, G. \& Coronado, E. Magnetic functionalities in MOFs: from the framework to the pore. Chem. Soc. Rev. 47, 533-557 (2018).

${ }^{81}$ Coronado, E., M. Giménez-Marqués, M., Mínguez Espallargas, G., Rey, F. \& Vitórica-Yrezábal, I. J. Spin-crossover modification through selective $\mathrm{CO}_{2}$ sorption. $J$. Am. Chem. Soc. 135, 15986-15989 (2013).

${ }^{82}$ Giménez-Marqués, M. et al. Gas confinement in compartmentalized coordination polymers for highly selective sorption. Chem. Sci. 8, 3109-3120 (2017).

${ }^{83}$ Muñoz, M. C. \& Real, J. A. Thermo-, piezo-, photo- and chemo-switchable metallocyanate based coordination polymers. Coord. Chem. Rev. 255, 2068-2093 (2011).

${ }^{84}$ Arcís-Castillo, Z. et al. Reversible chemisorption of sulfur dioxide in a spin crossover porous coordination polymer. Inorg. Chem. 52, 12777-12783 (2013).

${ }^{85}$ Rubio-Giménez, V. et al. High-quality metal-organic framework ultrathin films for electronically active interfaces. J. Am. Chem. Soc. 138, 2576-2584 (2016).

${ }^{86}$ Rubio-Giménez, V. et al. Bottom-up fabrication of semiconductive metal-organic framework ultrathin films. Adv. Mater. 30, 1704291 (2018).

${ }^{87}$ Rubio-Giménez, V. et al. Effect of the nanostructuration on the spin crossover transition in crystalline ultrathin films. Chem. Sci. 10, 4038-4047 (2019).

${ }^{88}$ Baldoví, J. J. et al. A SIM-MOF: three dimensional organization of single-ion magnets with anion-exchange capabilities. Chem. Eur. J. 20, 10695-10702 (2014).

${ }^{89}$ Aulakh, D. et al. Metal-organic frameworks as platforms for the controlled nanostructuring of single-molecule magnets. J. Am. Chem. Soc. 137, 9254-9257 (2015). 
${ }^{90}$ Abhervé, A. et al. Spin crossover encapsulation within a magnetic metal-organic framework. Chem. Commun., 52, 7360-7363 (2016).

${ }^{91}$ Clemente-León, M. et al. Insertion of a single-molecule magnet inside a ferromagnetic lattice based on a 3D bimetallic oxalate network: Towards molecular analogues of permanent magnets. Chem. Eur. J. 20, 1669-1676 (2014).

${ }^{92}$ Navarro-Moratalla, E. et al. Enhanced superconductivity in atomically thin $\mathrm{TaS}_{2}$. Nature Comm. 7, 11043 (2016).

${ }^{93}$ Huang, B. et al. Layer-dependent ferromagnetism in a van der Waals crystal down to the monolayer limit. Nature 546, 270-273 (2017).

${ }^{94}$ Huang, X. et al. Superconductivity in a Copper(II)-based coordination polymer with perfect Kagomé structure. Angew. Chem. Int. Ed. 57, 146-150 (2018).

${ }^{95}$ Abherbé, A., Mañas-Valero, S., Clemente-León, M. \& Coronado, E. Graphene related magnetic materials: micromechanical exfoliation of 2D layered magnets based on bimetallic anilate complexes with inserted $\left[\mathrm{Fe}^{\mathrm{III}}\left(\mathrm{acac}_{2} \text {-trien }\right)\right]^{+}$and $\left[\mathrm{Fe}^{\mathrm{III}}\left(\mathrm{sal}_{2} \text {-trien) }\right]^{+}\right.$ molecules. Chem. Sci. 6, 4665-4673 (2015).

${ }^{96}$ J. López Cabrelles, J. et al. Isoreticular two-dimensional magnetic coordination polymers prepared through pre-synthetic ligand functionalization. Nature Chem. 10, 1001-1007 (2018).

${ }^{97}$ Pedersen, K. S. et al. Formation of the layered conductive magnet $\mathrm{CrCl}_{2}$ (pyrazine) $)_{2}$ through redox-active coordination chemistry. Nature Chem. 10, 1056-1061 (2018).

${ }^{98}$ Suarez-Garcia, S. et al. Spin-crossover in an exfoliated coordination polymer and its implementation in thermochromic films. ACS Appl. Nano Mater. 1, 2662-2668 (2018).

${ }^{99}$ Dugay, J. et al. Phase transitions in spin-crossover thin fims probed by graphene transport measurements. Nano Lett. 17, 186-193 (2017).

${ }^{100}$ Wiesendanger, R. Nanoscale magnetic skyrmions in metallic films and multilayers: a new twist for spintronics. Nature Rev. Mater. 1, 16044 (2016).

${ }^{101}$ Zhang, B. et al. Quantum Spin Liquid from a three-dimensional copper-oxalate framework. J. Am. Chem. Soc. 140, 122-125 (2018).

${ }^{102}$ Liu, H. L. et al. Organic-based magnon spintronics. Nature Mater. 17, 308-312 (2018).

${ }^{103}$ Burch, K. S., Mandrus, D. \& Park, J.-G. Magnetism in two-dimensional van der Waals materials, Nature 563, 47-52 (2018).

${ }^{104}$ Someya, T., Bao, Z. N. \& Malliaras, G. G. The rise of plastic bioelectronics. Nature 540, 379-385 (2016).

${ }^{105}$ Giménez-Marqués, M., Hidalgo, T., Serre, C. \& Horcajada, P. Nanostructured metalorganic frameworks and their bio-related applications. Coord. Chem. Rev. 307, 342-360 (2016).

${ }^{106}$ A. Fert, A. Barthelemy, F. Petroff, Chapter 6 Spin transport in magnetic multilayers and tunnel junctions; Contemporary Concepts of Condensed Matter Science; Elsevier, 2006; Vol. 1, pp. 153-225.

${ }^{107}$ A. Barthelemy, A. Fert, J.-P. Contour, M. Bowen, V. Cros, J. M. De Teresa, A. Hamzic, J. C. Faini, J. M. George, J. Grollier, F. Montaigne, F. Pailloux, F. Petroff, C. Vouille, Magnetoresistance and spin electronics J Magn Magn Mater 2002, 242-245, 68.

${ }^{108} \mathrm{G}$. Tosi et al. Silicon quantum processor with robust long-distance qubit couplings.

Nature Commun. 8, 450 (2017)

${ }^{109} \mathrm{H}$. Bernien et al. Heralded entanglement between solid-state qubits separated by three metres. Nature 2013, 497, 86-90 
${ }^{110} \mathrm{M}$. Zhong et al. Optically addressable nuclear spins in a solid with a six hours coherence time. Nature 2015, 517, 177-180

${ }^{111}$ Astner et al. Solid-state electron spin lifetime limited by phononic vacuum modes. Nature Mater. 17, 313-317 (2018).

${ }^{112}$ C. Wang, D. Liu \& W. Lin. Metal-Organic Frameworks as a tunable platform for designing functional molecular materials. J. Am. Chem. Soc. 135, 13222-13234 (2013).

${ }^{113}$ Castro Neto A. H., Guinea, F., Peres, N. M. R., Novoselov, K. S. \& Geim, A. K. The electronic properties of graphene. Rev. Mod. Phys. 81, 109 (2009).

${ }_{114}$ Geim, A. K. Graphene: status and prospects. Science 324, 1530-1534 (2009).

115 Mannix, A. J., Kiraly, B., Hersam, M. C. \& Guisinger, N. P. Synthesis and chemistry of elemental 2D materials. Nat. Rev. Chem. 1, 0014 (2017).

${ }^{116}$ Duong, D. C., Yun, S. J. \& Lee, Y. H. Van der Waals layered materials: opportunities and challenges. ACS Nano 11, 11803-11830 (2017).

${ }^{117}$ Geim, A.K. \& Grigorieva, I.V. Van der Waals heterostructures. Nature 499, 419-425 (2013).

${ }^{118}$ Hirsch, A. \& Hauke, F. Post-Graphene 2D Chemistry: The Emerging Field of Molybdenum Disulfide and Black Phosphorus Functionalization. Angew. Chem. Int. Ed. 57, 4338-4354 (2018).

${ }^{119}$ Jariwala, D., Marks, T. J. \& Hersam, M. C. Mixed-dimensional van der Waals heterostructures. Nat.Mater. 16, 170-181 (2016).

${ }^{120}$ Xia, W. et al. Recent progress in van der Waals heterojunctions. Nanoscale 9, 43244365 (2017).

${ }^{121}$ Novoselov K. S., Mishchenko, A., Carvalho, A. \& Castro-Neto A. H. 2D materials and van der Waals heterostructures. Science 353, 9439 (2016).

${ }^{122} \mathrm{Li}$, C. et al. Engineering graphene and TMDs based van der Waals hetrerostructures for photovoltaic and photoelectrochemical solar energy conversion. Chem. Soc. Rev. 47, 4981-5037 (2018).

${ }^{123}$ Schwierz, F. Graphene transistors. Nature Nanotech. 5, 487 (2010).

${ }^{124}$ Huang, X., Qi, X., Boey, F. \& Zhang, H. Graphene-based composites. Chem. Soc. Rev. 41, 666 (2012).

\section{Acknowledgements}

This work is dedicated to Professor Jean Paul Malrieu in occasion of his 80 birthday. Most of the examples reported in this article to illustrate my personal vision on the past, present and future of the Molecular Magnetism have been taken from the work of my group. I am greatly indebted to all of them for continuous support and fruitful discussions. I would like also to thank all the coworkers and colleagues who have contributed, each in their own way, to the development of the field in the last years. Their names appear in the references. Financial support from the European Union (COST Action MolSpin 15128 on Molecular Spintronics and ERC Advanced Grant Mol-2D 788222), the Spanish MINECO (grants MAT2017-89993-R, cofinanced by FEDER, and Excellence Unit María de Maeztu MDM-2015-0538) and the Generalitat Valenciana (Prometeo Programme of Excellence) is gratefully acknowledged. 


\section{FIGURES}
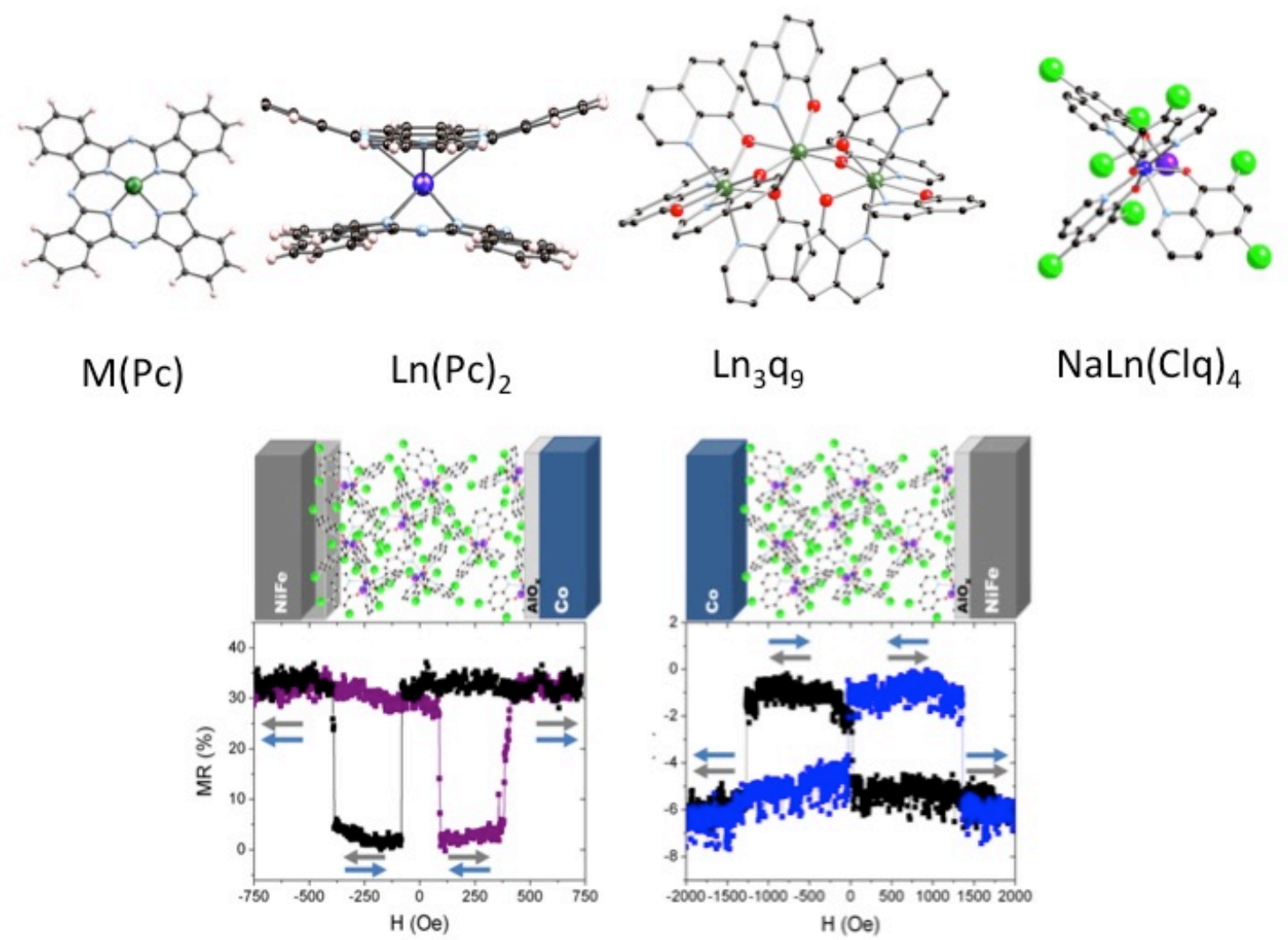

Figure 1. Top: Examples of thermally stable magnetic molecules used in spintronics: MPc's, $\operatorname{Ln}(\mathrm{Pc})_{2}, \mathrm{Ln}_{3} \mathrm{q}_{9}, \mathrm{NaLnq}_{4}$. Bottom: Spinterface effects in the molecular spin valve $\mathrm{NiFe} / \mathrm{NaDyClq} / \mathrm{Co}$, where NaDyClq refers to a mononuclear Dy(III) complex based on a chlorine-di-substituted quinoline ligand. The sign in the magnetoresistance is positive if an $\mathrm{AlO}_{\mathrm{x}}$ barrier is inserted between the $\mathrm{NiFe}$ electrode and the molecule (right), but it changes to negative when the $\mathrm{AlO}_{\mathrm{x}}$ layer is placed on the Co electrode (left). 

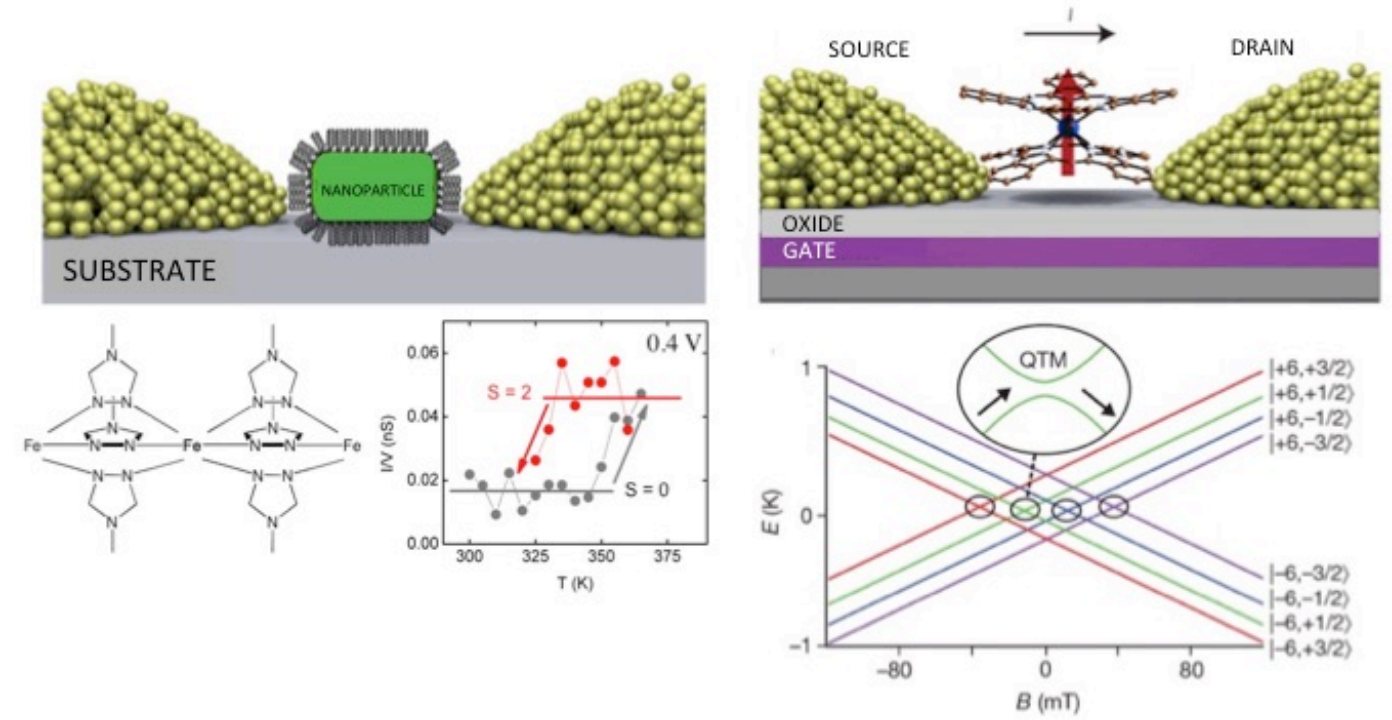

Figure 2. Left: Spin-switching in electronic devices formed by individual spincrossover $\mathrm{Fe}(\mathrm{II})$ nanoparticles. Right: Electrical read-out and manipulation of the nuclear spin of a single bis-phtalocyanine $\mathrm{Tb}^{+3}$ molecule. For certain values of the magnetic field the electronic states are crossing and the molecule can reverse its magnetic moment. At these points a change in the conductance is observed, which allows to detect the nuclear spin state of $\mathrm{Tb}^{+3}$. 

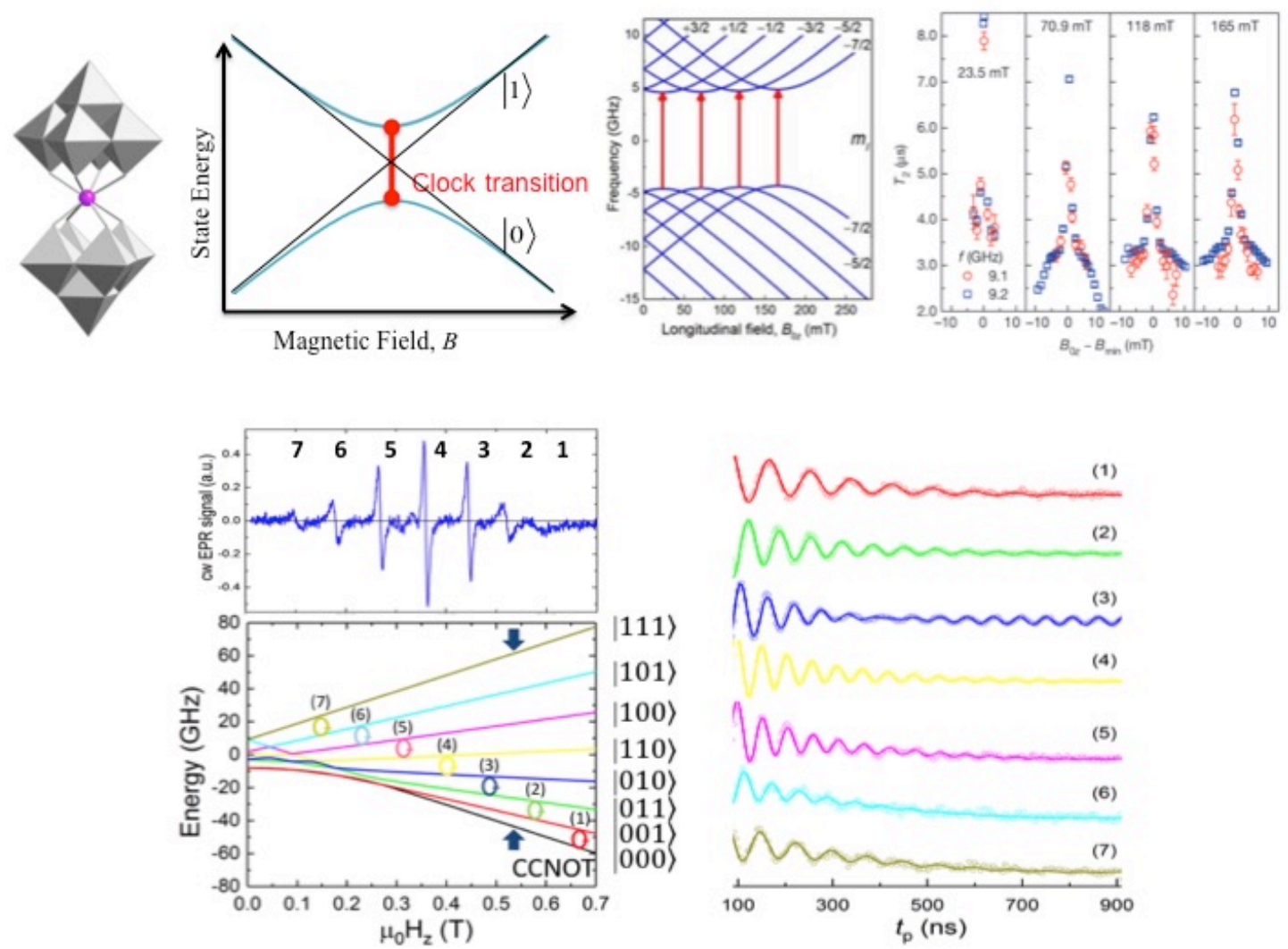

Figure 3. Top: Enhancing quantum-coherence in the POM molecule $\left[\mathrm{HoW}_{10} \mathrm{O}_{36}\right]^{9-}$ (left) via atomic clock transitions (centre). At the four magnetic fields in which the clock transitions are located, the $T_{2}$ values show a divergence (right). Bottom: Example of a three-qubit processor based on the $\mathrm{Gd}^{+3} \mathrm{POM}$ molecule $\left[\mathrm{Gd}\left(\mathrm{H}_{2} \mathrm{O}\right) \mathrm{P}_{5} \mathrm{~W}_{30} \mathrm{O}_{110}\right]^{12-}$. Its spin multiplet $(S=7 / 2)$ has 8 spin sublevels and 7 EPR active transitions (left) connecting these sublevels. These transitions are coherent and addressable through X-band pulsed EPR (right). 

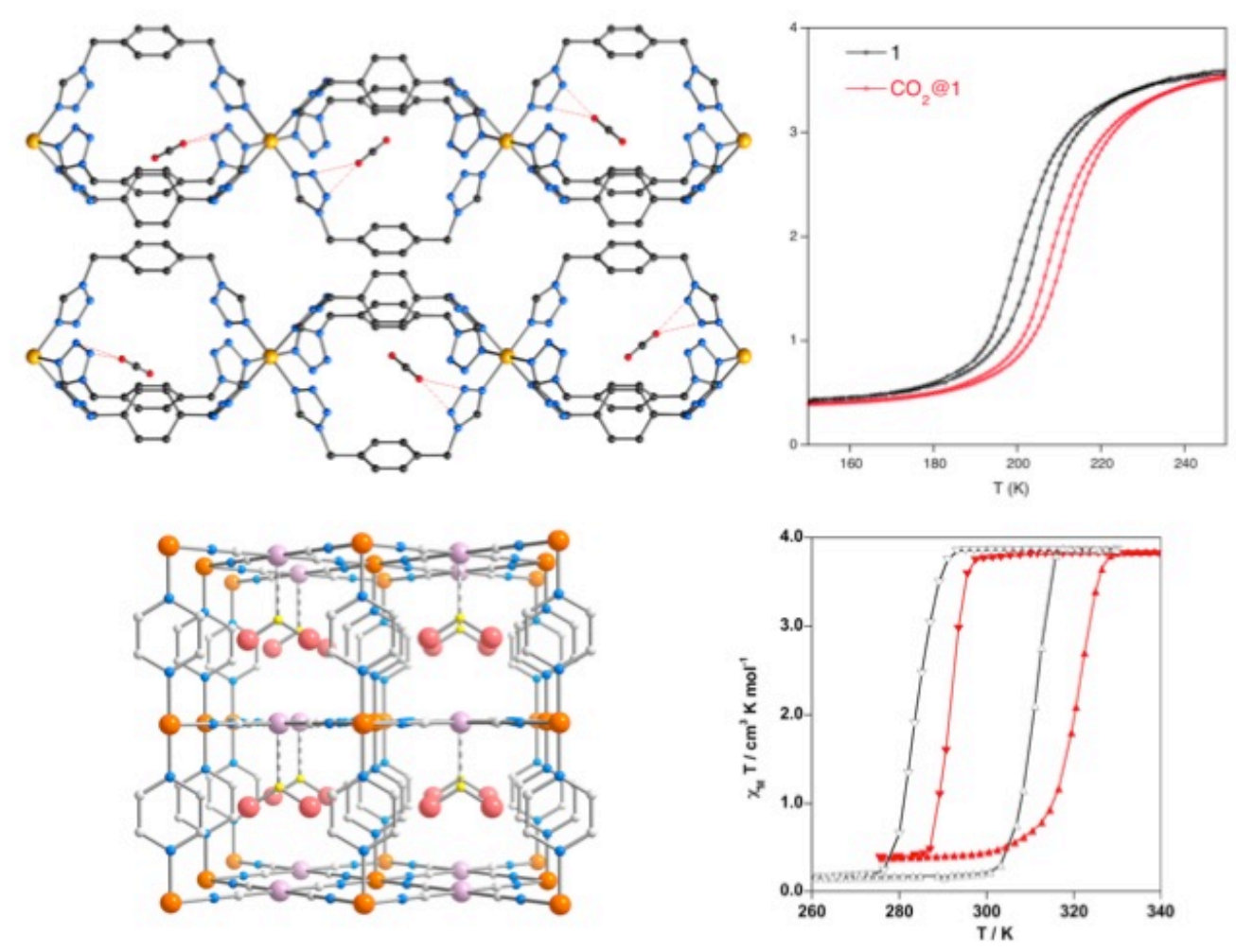

Figure 4. SCO-containing MOFs. Top: Stimuli-responsive magnetic MOFs based on spin-crossover $(\mathrm{SCO})$ complexes $\left[\mathrm{Fe}(\mathrm{btzx})_{3}\right](\mathrm{X})_{2}$ and $\left[\mathrm{Fe}(\mathrm{btzx})_{3}\right]\left(\mathrm{BF}_{4}\right)_{2} \quad(\mathrm{btzx}=$ bistetrazol- $p$-xylene; $\mathrm{X}=\mathrm{ClO}_{4}{ }^{-}$and $\mathrm{BF}_{4}^{-}$) showing the trapping of $\mathrm{CO}_{2}$ in the cavities (left) and the effect of this trapping on the magnetic properties (right). Bottom: SCO Hoffmann clathrates: (left) Structure of $\left\{\mathrm{Fe}(\mathrm{pz})\left[\mathrm{Pt}^{\mathrm{II}}(\mathrm{CN})_{4}\right]\right\} \cdot \mathrm{SO}_{2}$; (right) Magnetic properties of $\left\{\mathrm{Fe}(\mathrm{pz})\left[\mathrm{Pt}^{\mathrm{II}}(\mathrm{CN})_{4}\right]\right\}$ (black line) and $\left\{\mathrm{Fe}(\mathrm{pz})\left[\mathrm{Pt}^{\mathrm{II}}(\mathrm{CN})_{4}\right]\right\} \cdot \mathrm{SO}_{2}$ (red line). 

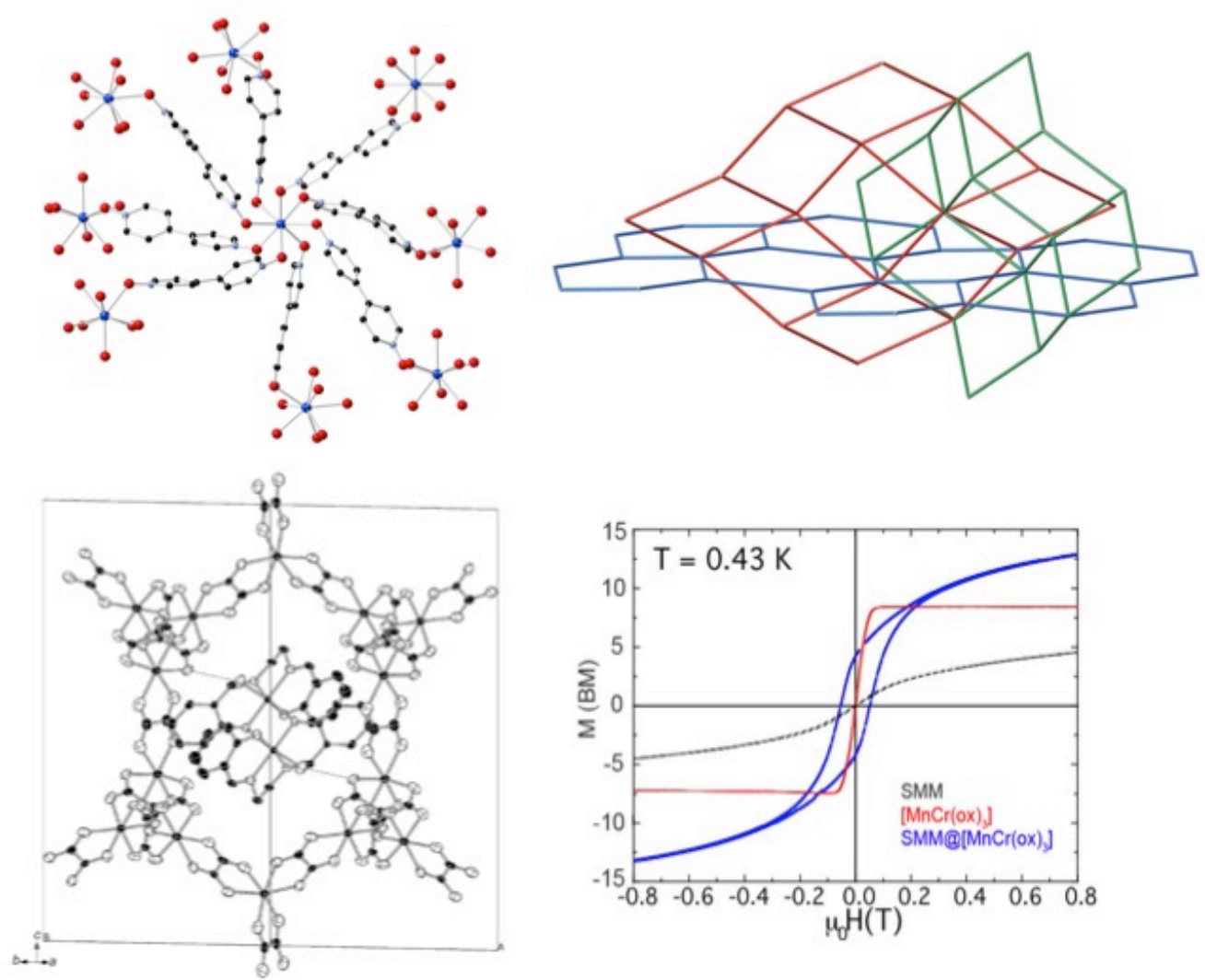

Figure 5. SMM-containing MOFs. Top: Coordination polymers containing lanthanoid single-ion magnets in the nodes of a porous framework formed by bipyNO as bridging ligand. The complex metallic framework, comprising three independent nets, is shown on the right. Bottom: Hybrid magnets formed by a ferromagnetic MOF filled with SMMs in the pores. The interaction between the two magnetic components leads to a drastic enhancement in the magnetic coercivity of these hybrid magnets (right). 

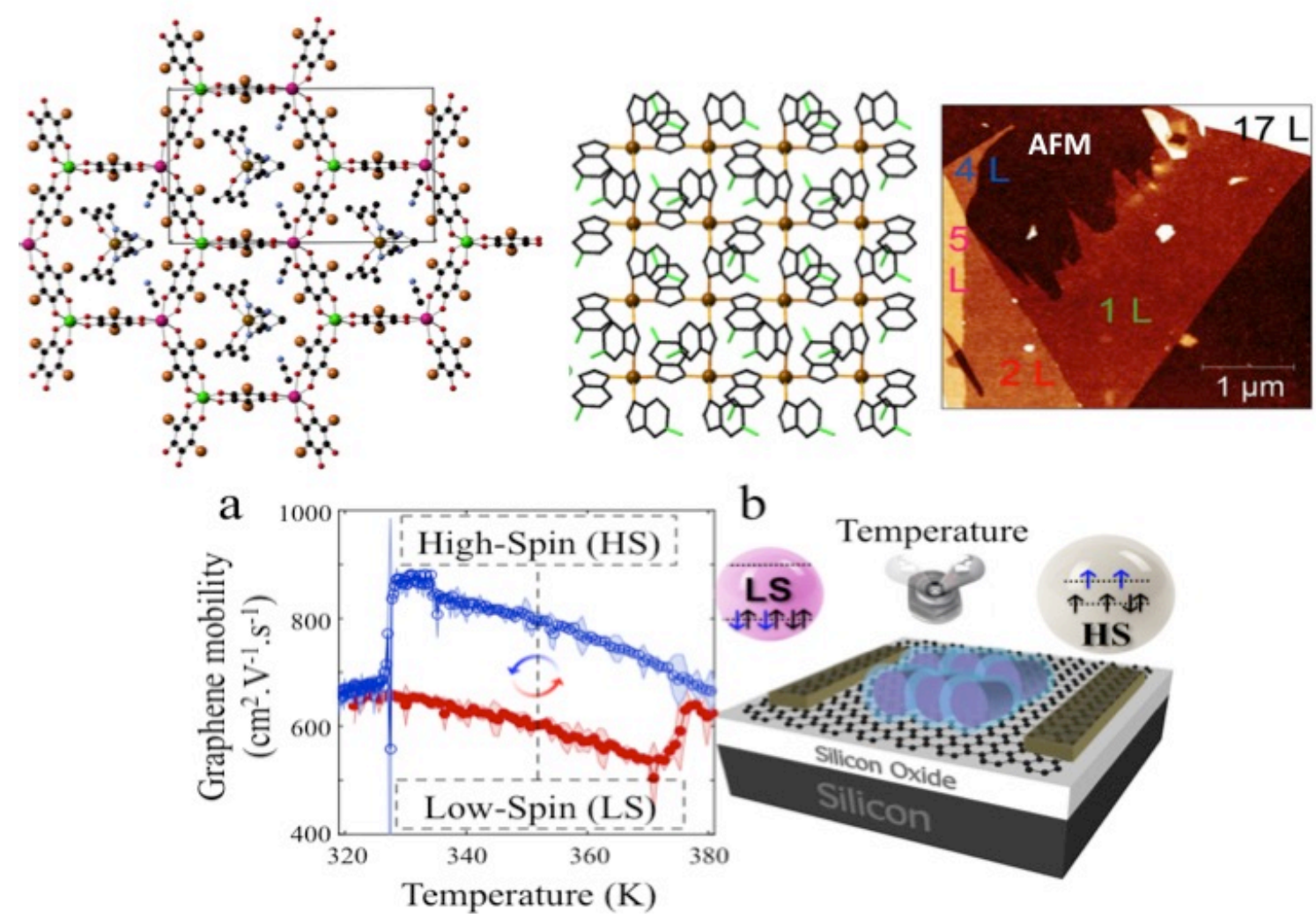

Figure 6. Top: 2D magnetic layers based on coordination polymers: left) an anionic honeycomb framework, based on a bimetallic $\mathrm{Mn}^{\mathrm{II}} \mathrm{Cr}^{\mathrm{III}}$ anilate complex, filled with $\left[\mathrm{Fe}^{\mathrm{III}}\left(\mathrm{acac}_{2} \text {-trien }\right)\right]^{+}$counter-ions in the pores; centre) a neutral square lattice formed by distorted tetrahedral $\mathrm{Fe}^{\mathrm{II}}$ centers in the nodes of the layer and bridged by benzimidazoletype ligands; right) AFM image showing exfoliated single layers of this layered material. Bottom: Hybrid molecular/2D heterostructures formed by a layer of SCO nanoparticles deposited on graphene showing how graphene is sensing the spin state of the SCO layer. 